\title{
Finite speed of propagation for the thin-film equation and other higher-order parabolic equations with general nonlinearity
}

\author{
DANIELE ANDREUCCI ${ }^{\dagger}$ \\ Dipartimento Metodi e Modelli, Università La Sapienza, via A.Scarpa 16, 00161 Roma, Italy \\ AND \\ ANATOLI F. TEDEEV \\ Institute of Applied Mathematics, Academy of Sciences, R.Luxemburg st.74, 340114 Donetsk, \\ Ukraine
}

[Received 11 November 1999 and in revised form 15 January 2001]

\begin{abstract}
We prove the property of finite speed of propagation for degenerate parabolic equations of order $2 m \geqslant 2$, when the nonlinearity is of general type, and not necessarily a power function. We also give estimates of the growth in time of the interface bounding the support of the solution.

In the case of the thin-film equation, with non-power nonlinearity, we obtain sharp results, in the range of nonlinearities we consider. Our optimality result seems to be new even in the case of power nonlinearities with general initial data.

In the case of the Cauchy problem for degenerate equations with general $m$, our main assumption is a suitable integrability Dini condition to be satisfied by the nonlinearity itself. Our results generalize Bernis' estimates for higher-order equations with power structures. In the case of second-order equations we also prove $L^{\infty}$ estimates of solutions.
\end{abstract}

\section{Introduction}

We consider in this paper two problems for degenerate higher-order parabolic equations. Our main result is an estimate of the finite speed of propagation of compactly supported solutions to these problems.

Let us first introduce an initial-value boundary problem for the well known thin-film equation arising in the theory of lubrication, and studied both for its mathematical and physical interest. We consider the equation

$$
u_{t}+\left(f(|u|) u_{x x x}\right)_{x}=0
$$

set in the domain $(x, t) \in Q_{T}=(-R, R) \times(0, T), R, T>0$, together with the initial and boundary conditions

$$
\begin{array}{rlrlrl}
u(x, 0) & =u_{0}(x), & & & -R<x<R, \\
u_{x}(-R, t) & =0, & u_{x}(R, t) & =0, & & 0<t<T, \\
u_{x x x}(-R, t) & =0, & u_{x x x}(R, t) & =0, & & 0<t<T .
\end{array}
$$

Email: andreucc@dmmm.uniroma1.it 
The case we cover is the one corresponding to the range $2<n<3$ for $f(s)=s^{n}$, which is known in the literature $[10,15,17,24]$. Our proof provides an alternative approach in the case of power nonlinearities, and is of more general scope: see Section 6 for the precise assumptions we stipulate on $f$. For example, functions like the ones in (1.12) below (for $s>0$ ) are admissible choices of $f$, provided $3>a>2, q \in \mathbb{R}$. Sums of suitable power functions are also admissible: see [16] for references.

We consider suitable non-negative solutions of the problem at hand, i.e. solutions which can be approximated by positive regular solutions (see Section 6 for details). We prove that such solutions have the property of finite speed of propagation and, more exactly, that if supp $u_{0} \subset\left[-r_{0}, r_{0}\right]$, then $\operatorname{supp} u(\cdot, t) \subset[-z(t), z(t)]$ for large $t>0$, where $z(t)$ is defined by (setting $\Omega=(-R, R)$ )

$$
\frac{z(t)^{4}}{t}=C f\left(\frac{\left\|u_{0}\right\|_{1, \Omega}}{z(t)}\right) .
$$

Here $C$ depends only on certain structural properties of $f$ : see Theorem 6.1 for a precise statement. Note that this estimate is optimal, as we prove in Proposition 6.1; this seems to be a new result (for general initial data) even in the case of power nonlinearities (the case of source-type solutions was treated in [17]).

We do not attempt to give here an account of the large literature in the field of the thin-film equation; we refer the reader to the papers mentioned above, and to [19] and [18] for an interesting discussion of the positivity properties of solutions to (1.1) and related equations.

We have collected all the material concerning the problem for the thin-film equation in Section 6, as we think this makes its presentation clearer. The rest of this Introduction and Sections 2-5 are devoted to our second problem, (1.5), (1.6). Actually, we apply to both problems essentially the same method: that is an energy approach relying on the embedding inequality we prove in Section 3 . The method is indeed flexible enough to cover equations of form (1.5) of any order, and even equations with a different structure, like (1.1).

More precisely, the main ideas of the proofs of the finite speed of propagation property (proof of Theorem 1.1 in Section 4 for problem (1.1)-(1.4), and proof of Theorem 6.1 in Section 6 for problem (1.5), (1.6)) are (1) obtaining a suitable energy inequality (see (4.5) and (6.22)) in a sequence of expanding domains, (2) applying a new embedding result for non-power functions (i.e. Lemma 3.1) to obtain an 'improved form' of the energy inequality (see (4.7) and (6.27)) and (3) using the latter to construct an iteration process, finally showing that a certain integral norm of $u$ (and therefore $u$ itself) vanishes on annuli $\{\rho / 2<|x|<\rho\}$ if $\rho$ is large enough (see (4.8)-(4.10) and (6.28), (6.29)).

Let us introduce the Cauchy problem

$$
\begin{aligned}
b(u)_{t}+(-1)^{m} \sum_{|\alpha|=m} D^{\alpha}\left(\left|D^{m} u\right|^{p-2} D^{\alpha} u\right) & =0, & & \text { in } \mathbb{R}^{N} \times(0, \infty), \\
b(u(x, 0)) & =v_{0}(x), & & \text { in } \mathbb{R}^{N},
\end{aligned}
$$

where $N, m \geqslant 1$ are integers, and $p>1$. The initial data $v_{0} \in L^{1}\left(\mathbb{R}^{N}\right)$ will be required later to satisfy, when $m>1$, certain additional integrability conditions.

For general $m$ we investigate the property of finite speed of propagation, but, in the case of problems of second order, i.e. $m=1$, we establish both sharp $L^{\infty}$ bounds of solutions and optimal estimates of the speed of propagation of the support. To the best of our knowledge, the property of finite speed of propagation under the Dini condition (1.7) in the multidimensional case $N>1$ has not been proven before, with the exception of the papers $[20,35]$ which treat just the case $m=1$, 
$p=2$ with methods based on the comparison principle. Our methods rely instead on integral estimates, and are of more general scope. In fact Theorems 1.1 and 1.2 below apply to solutions of equations of more general form, modelled after (1.5). This is an obvious consequence of the proofs given here. See Section 2 for an approach based on comparison with an explicit supersolution.

When $m=1$, the second-order equation (1.5) appears in modelling the flow of a gas through a porous medium (see the references in [23]); it is sometimes referred to, in this connection, as the equation of non-Newtonian elastic filtration (the Newtonian case being the one with $p=2$ ). Actually, owing to the definition of $\left|D^{m} u\right|$ we give below, (1.6) does not reduce exactly to the $p$ Laplacian equation when $m=1$ and $b(u)=u$, but this standard operator can be treated without any change in our approach: see Remark 1.6. A great deal of work has been attracted by this subject in the past (see $[23,26]$ and references therein). It seems that our results are new even in the case $m=1$.

Note that in (1.5) the sum is extended to all the space derivatives of order $m$. Moreover, we use the notation

$$
\left|D^{j} u\right|=\left(\sum_{|\alpha|=j}\left|D^{\alpha} u\right|^{p}\right)^{1 / p}, \quad\left\|D^{j} u\right\|_{p, \mathbb{R}^{N}}=\left(\int_{\mathbb{R}^{N}}\left|D^{j} u(x)\right|^{p} \mathrm{~d} x\right)^{1 / p}, \quad 0 \leqslant j \leqslant m .
$$

We assume that $b$ and its inverse function $\beta=b^{-1}$ are increasing functions, of class $A C_{\text {loc }}$ in $\mathbb{R}$, with $b(0)=0$. Our main assumption is

$$
\mathcal{D}(s):=\int_{0}^{s} \frac{\mathrm{d} \tau}{b(\tau)^{\frac{1}{p-1}}}<\infty, \quad s \in \mathbb{R},
$$

where we let $r^{a}=|r|^{a-1} r$ for $r \neq 0, a \in \mathbb{R}$. In fact, this sole assumption is sufficient to imply finite speed of propagation for solutions of (1.5), at least if $m=1$ : see Section 2. We also need for technical reasons (with the exception of Section 2) the assumptions

$$
\beta^{\prime}(s)|s| \leqslant v|\beta(s)|, \quad \text { a.e. } s \in \mathbb{R},
$$

for some $v>0$, and

$$
\mathcal{D}(s) \leqslant c\left(\frac{s}{s_{0}}\right)^{\lambda} \mathcal{D}\left(s_{0}\right),
$$

for all $s, s_{0}>0, s \leqslant s_{0}$; here $c, \lambda>0$ are given.

In order to find sharper estimates, we need additionally

$$
\beta^{\prime}(s)|s| \geqslant \mu|\beta(s)|, \quad \text { a.e. } s \in \mathbb{R},
$$

for some $\mu>0$. In fact, the parameters $\nu, \lambda$, and $\mu$ when (1.10) is stipulated, do not enter the functional form of our estimates, which is determined only by the function $b$, and by $N, m$, $p$, of course.

REMARK 1.1 In fact (1.10) is connected with (1.9): if we assume $\mu(p-1)>1$ above, one can check that (1.9) holds (see also (3.9) below). 
An equivalent form of (1.9) is

$$
\mathcal{D}(\alpha s) \leqslant \alpha^{\prime} \mathcal{D}(s), \quad \text { for all } s>0,
$$

for some $\alpha, \alpha^{\prime} \in(0,1)$.

For the sake of notational simplicity, we that assume throughout

$$
c_{1}|b(-s)| \geqslant b(s) \geqslant c_{1}^{-1}|b(-s)|, \quad \text { for a given } c_{1}>1 \text { and all } s>0 .
$$

If this assumption is violated, our results still hold, though the corresponding statements are formally more involved. For example, the function $\varphi$ in (1.20) below should be replaced with $\max (\varphi(s), \varphi(-s))$, and so on. One should also rephrase (1.9) for both positive and negative $s$.

REMARK 1.2 Power functions $b(s)=s|s|^{a-1}, 0<a<p-1$, are examples of admissible nonlinearities. Further examples are provided by functions given by

$$
b(s)=s|s|^{a-1}|\ln | s||^{q},
$$

for $0<s \ll 1$ and for $s \gg 1$ (and suitably extended for intermediate values of $s$ ). Indeed, when $0<a<p-1$ and $q \in \mathbb{R}$ is arbitrary, such functions satisfy all our assumptions. Note that $b$ given as in (1.12) (for small $s$, of course) satisfies the Dini condition (1.7) even if $a=p-1, q>p-1$. Thus Section 2 remains valid in this case.

Let us introduce the non-negative functions

$$
\Phi(s)=\int_{0}^{b(s)} \beta(\tau) \mathrm{d} \tau, \quad \varphi(s)=\int_{0}^{s} b(\tau) \mathrm{d} \tau, \quad s \in \mathbb{R} .
$$

We denote by $\gamma, \gamma_{0}$ generic positive constants depending on $N, m, p$ and on the constants appearing in the assumptions above.

DEFINITION 1.1 We say that $u$ is an energy solution of (1.5), (1.6) if $u \in L^{p}(0, T$; $W^{m, p}\left(\mathbb{R}^{N}\right)$ ), for all finite $T>0$, and

$$
\int_{0}^{\infty} \int_{\mathbb{R}^{N}}\left\{-b(u) \zeta_{t}+\sum_{|\alpha|=m}\left|D^{m} u\right|^{p-2} D^{\alpha} u D^{\alpha} \zeta\right\} \mathrm{d} x \mathrm{~d} t=\int_{\mathbb{R}^{N}} v_{0}(x) \zeta(x, 0) \mathrm{d} x,
$$

for all $\zeta \in C^{m}\left(\mathbb{R}^{N} \times[0, \infty)\right)$, such that $\operatorname{supp} \zeta$ is bounded.

Moreover, we stipulate that $\left\|\Phi\left(u_{0}\right)\right\|_{1, \mathbb{R}^{N}}<+\infty$, where $u_{0}=\beta\left(v_{0}\right)$, and that it is possible to integrate by parts so as to get (formally multiplying the equation by $u \zeta$ )

$$
\begin{aligned}
\int_{\mathbb{R}^{N}} \Phi(u(x, t)) \zeta(x) \mathrm{d} x+\sum_{|\alpha|=m} \int_{0}^{t} \int_{\mathbb{R}^{N}} D^{\alpha}(u \zeta) D^{\alpha} u\left|D^{m} u\right|^{p-2} \mathrm{~d} x \mathrm{~d} \tau & \\
& =\int_{\mathbb{R}^{N}} \Phi\left(u_{0}(x)\right) \zeta(x) \mathrm{d} x,
\end{aligned}
$$

for a $\zeta=\zeta(x) \geqslant 0, \zeta \in C_{0}^{m}\left(\mathbb{R}^{N}\right)$, for almost all $t>0$. (In Remark 1.5 we point out how in some cases this last assumption about integration by parts can be removed.) 
DEFINITION 1.2 We say that $u$ is a weak solution of (1.5), (1.6) with $m=1$ if it is locally bounded in $\mathbb{R}^{N} \times(0, \infty), u \in L_{\mathrm{loc}}^{p}\left(0, \infty ; W^{m, p}\left(\mathbb{R}^{N}\right)\right)$, and (1.14) is satisfied. In the same spirit of Definition 1.1, we assume a priori that we can carry out integration by parts as in (5.1) and in (5.13) below.

REMARK 1.3 It follows from Definition 1.1 that we can multiply (1.5) by $u$ and integrate by parts, proving

$$
\|\Phi(u(t))\|_{1, \mathbb{R}^{N}} \leqslant\left\|\Phi\left(u_{0}\right)\right\|_{1, \mathbb{R}^{N}}, \quad \text { for all } t>0 .
$$

Let us also remark that, if both (1.8) and (1.10) are stipulated, we have

$$
(1+v)^{-1} \varphi(s) \leqslant \Phi(s) \leqslant(1+\mu)^{-1} 2^{1+\frac{1}{\mu}} \varphi(s) .
$$

These inequalities can be proven by integrating by parts the integral defining $\Phi(s)$. In order to prove the second bound in (1.17), we also use the first of (3.7) below to get $s b(s) \leqslant 2^{1+1 / \mu} \varphi(s)$. Therefore, (1.16) can be restated in terms of $\varphi$.

If we define $v=b(u)$, then $v$ solves, formally,

$$
v_{t}+(-1)^{m} \sum_{|\alpha|=m} D^{\alpha}\left(\left|D^{m} \beta(v)\right|^{p-2} D^{\alpha} \beta(v)\right)=0, \quad \text { in } \mathbb{R}^{N} \times(0, \infty) .
$$

The precise definition of solution in terms of $v$ follows trivially from the definitions above. This formulation is used in Sections 2 and 5.

Let us define

$$
Z(t)=\inf \{r>0 \mid u(x, t)=0 \text { a.e. }|x|>r\}, \quad t \geqslant 0 .
$$

Our main results are the following two theorems.

THEOREM 1.1 Assume that (1.7)-(1.9) and (1.11) hold. Let $u$ be an energy solution of the problem (1.5), (1.6), with compactly supported initial data $v_{0}$, so that supp $v_{0} \subset B_{r_{0}}$, for some $1<r_{0}<\infty$. Then

$$
Z(t) \leqslant \gamma_{1} r_{0}+\gamma_{2} t^{\frac{1}{m p}}, \quad t>0,
$$

where $\gamma_{2}$ depends also on $\left\|\Phi\left(u_{0}\right)\right\|_{1, \mathbb{R}^{N}}$.

If, in addition, we stipulate also (1.10), then we have the sharper estimate

$$
Z(t)^{N} \varphi\left(\mathcal{D}^{-1}\left[\left(\frac{Z(t)^{m p}}{t}\right)^{\frac{1}{p-1}}\right]\right) \leqslant \gamma\left\|\varphi\left(u_{0}\right)\right\|_{1, \mathbb{R}^{N}},
$$

for $t>\bar{t}\left(r_{0},\left\|\varphi\left(u_{0}\right)\right\|_{1, \mathbb{R}^{N}}\right)$.

THEOREM 1.2 Let $m=1$, and let $u$ be a weak solution of (1.5), (1.6). Assume (1.7), (1.8), (1.10), (1.11), with $\mu(p-1)>1, \mu \geqslant 1$. Then

$$
b\left(\|u(\cdot, t)\|_{\infty, \mathbb{R}^{N}}\right) \mathcal{D}\left(\|u(\cdot, t)\|_{\infty, \mathbb{R}^{N}}\right)^{\frac{N}{p}(p-1)} \leqslant \gamma t^{-\frac{N}{p}}\left\|v_{0}\right\|_{1, \mathbb{R}^{N}}, \quad t>0 .
$$

Moreover, if we assume that supp $v_{0} \subset B_{r_{0}}, r_{0}>1$, then for $t>\bar{t}\left(r_{0},\left\|v_{0}\right\|_{1, \mathbb{R}^{N}}\right)$,

$$
\gamma_{0}\left|\int_{\mathbb{R}^{N}} v_{0}(x) \mathrm{d} x\right| \leqslant Z(t)^{N} b\left(\mathcal{D}^{-1}\left[\left(\frac{Z(t)^{p}}{t}\right)^{\frac{1}{p-1}}\right]\right) \leqslant \gamma\left\|v_{0}\right\|_{1, \mathbb{R}^{N}} .
$$


Note that the bound for $Z(t)$ in (1.22) is sharp, at least if $\int_{\mathbb{R}^{N}} v_{0} \neq 0$.

REMARK 1.4 (Power NONLINEARities) If $b(s)=s^{a}$, that is $\beta(s)=s^{\alpha}, \alpha=1 / a$, for $s \in \mathbb{R}$, and a given $a<p-1$, estimate (1.20) becomes

$$
Z(t) \leqslant \gamma\left\|v_{0}^{\alpha+1}\right\|_{1, \mathbb{R}^{N}}^{\frac{p-1-a)}{N(1-a)+m p(a+1)}} t^{\overline{\alpha(p-1-a)+m p(a+1)}}, \quad t>\bar{t},
$$

which is the bound given in [12]. In fact, the equations considered in [12], though slightly different from (1.5), fit in the class described in Remark 1.6 below.

When $m=1,(1.21)$ and (1.22) reduce to the known estimates

$$
\begin{aligned}
\|u(\cdot, t)\|_{\infty, \mathbb{R}^{N}} \leqslant \gamma\left\|v_{0}\right\|_{1, \mathbb{R}^{N}}^{\frac{p}{N(p-a)+p a}} t^{-\frac{N}{N(p-1-a)+p a}}, \quad t>0, \\
Z(t) \leqslant \gamma\left\|v_{0}\right\|_{1, \mathbb{R}^{N}}^{\frac{p-1-a)+p a}{N(1-a)}} t^{\frac{a}{N(p-1-a)+p a}}, \quad t>\bar{t} .
\end{aligned}
$$

In the case of power nonlinearities the proof of Theorem 1.2 is considerably easier than in the general case, as we point out in Remark 5.1.

REMARK 1.5 (INTEGRATION BY PARTS) It is known that, when dealing with general nonlinearities, or with higher-order equations, the problem of integration by parts is not trivial: see $[1,11,12]$. However, one can check, with the help of the classical results of [31], that the assumptions of Proposition 3.1 in [12] are in fact satisfied in our case if we take $X=W^{m, p}\left(\mathbb{R}^{N}\right) \cap L^{\varphi}\left(\mathbb{R}^{N}\right)$ and

$$
Y=L^{\varphi}\left(\mathbb{R}^{N}\right)=\left\{f \mid \int_{\mathbb{R}^{N}} \varphi(f(x)) \mathrm{d} x<+\infty\right\},
$$

provided we assume, in order to fit simply in the theory of [31], that $b$ is an odd function satisfying (1.8), (1.10). In $Y$ we define the usual Orlicz norm. Therefore, at least in this case, we can in fact prove the formula of integration by parts in Definition 1.1, and consequently the bound (1.16) for $\Phi(u(t))$ in $L^{1}\left(\mathbb{R}^{N}\right)$, rather then including it in the definitions.

Under the same assumptions, existence of energy solutions follows from the energy estimate, and from Bernis' approach in [11].

REMARK 1.6 Our results and techniques apply to equations in the more general form

$$
b(u)_{t}+(-1)^{m} \sum_{|\alpha|=m} D^{\alpha} A_{\alpha}(x, u, \tilde{D} u)=0,
$$

where $\tilde{D} u$ denotes the vector whose components are all the space derivatives of $u$ of order less than or equal to $m$. We have here to assume the conditions

$$
\sum_{|\alpha|=m}\left|A_{\alpha}(x, u, \tilde{D} u)\right| \leqslant c_{0}\left|D^{m} u\right|^{p-1}, \quad \sum_{|\alpha|=m} A_{\alpha}(x, u, \tilde{D} u) D^{\alpha} u \geqslant c_{1}\left|D^{m} u\right|^{p},
$$

with two suitable constants $c_{0}, c_{1}>0$.

A further example is provided by equations of the type

$$
b(u)_{t}+\Delta^{\frac{m}{2}}\left(\left|\Delta^{\frac{m}{2}} u\right|^{p-2} \Delta^{\frac{m}{2}} u\right)=0
$$

under the same assumptions as above (but here $m \geqslant 2$ is even). The proof stays unchanged in this case, after an application of the Calderon-Zygmund inequality (see Remark 4.1 below; see also Section 11.3 in [12] for a different approach when $b(u)=u$ ). 
In the case of power functions $b(s)=s^{a}$, the property of finite speed of propagation, that is $Z(t)<$ $\infty$ if $Z(0)<\infty$, was shown to hold in [7] for $m=1$, and for any $N \geqslant 1$, provided $a<p-1$. For all $m \geqslant 1$ and for all $N \geqslant 1$ the result was proven in the breakthrough paper [12]. See also [13] for an exposition on the application of local energy methods to problems of this kind (the equation of thin films is treated in $[14,15])$. In the case of general nonlinearities, and $p=2, N=1, m=1$, finite speed of propagation was proven in [27] under the Dini condition (1.7), which was proved to be also necessary in $[25,30]$. Finally, it has been recently proved in [23] that condition (1.7) is necessary and sufficient for the property of finite speed of propagation to hold, in the case $N=1$, $m=1$, and for general $p$ (the authors treated even more general equations involving lower-order terms). We refer the reader to the paper [23] for further information on the literature dealing with this problem.

In the case $N>1$, the use of energy methods in the proof of finite speed of propagation dates back to [6,21]. Our method relies on local energy estimates and, when $m=1$, on sup bounds of the solution, which yield optimal results, as pointed out above. In the case $m=1$, the growth of $Z$ is estimated only in terms of the $L^{1}$ norm of the initial data $v_{0}$. Our technique is similar to the one developed in $[4,5]$ to obtain sharp bounds in domains different from $\mathbb{R}^{N}$ (for power nonlinearities). However, when $m>1$ we cannot exploit the $L^{\infty}$ bounds as we did there. The case when $b$ is a power function has also been treated in $[32,33]$, with a method based on integral estimates, but completely different from ours.

Structure of the paper. Section 2 is devoted to the construction of an explicit supersolution to (1.5). Section 3 contains the embedding result that is central to our approach. In Sections 4, and 5, we prove Theorems 1.1 and 1.2, respectively. Finally, Section 6 deals with the thin-film equation.

\section{An explicit supersolution}

If $m=1$ and the structure of the equation is exactly the one in (1.5), we can prove finite speed of propagation of solutions corresponding to compactly supported and bounded initial data just assuming (1.7). This is done via comparison results similar to the ones in [9], which are applicable [8] to the supersolution of (1.5) defined below. Indeed, we work here with the formulation of the problem in terms of $v=b(u)$, and following ideas similar to the ones developed in [21,35] when $p=2$.

Note that

$$
\mathcal{D}(\beta(s))=\int_{0}^{s} \frac{\beta^{\prime}(\tau)}{\tau^{\frac{1}{p-1}}} \mathrm{~d} \tau,
$$

and define the function $V(x, t)$ by means of

$$
\mathcal{D}(\beta(V))=\xi, \quad \text { where } \quad \xi(x, t)=\left[a-c(p) \frac{|x|^{\frac{p}{p-1}}}{(t+d)^{\frac{1}{p-1}}}\right]_{+},
$$

and $c(p)=(p-1) p^{-p /(p-1)}$. Here $a$ and $d$ are two arbitrary positive constants. For each $t>0$, the support of $V(\cdot, t)$ is bounded, and coincides with the support of $\xi(\cdot, t)$. Moreover, we can check that, at least formally,

$$
V_{t}-\operatorname{div}\left(|D \beta(V)|^{p-2} D \beta(V)\right)=\frac{1}{p} \frac{N V}{t+d} \geqslant 0, \quad \text { in } \mathbb{R}^{N} \times(0, \infty) .
$$


In fact, elementary calculations show that $V$ is a weak solution of (2.1) in the sense of Definition 1.2 (with obvious modifications), and the quoted comparison principle yields the result.

\section{Preliminary results. Embedding}

First, we gather here, for the reader's convenience, some elementary facts following from our assumptions.

It follows by integration from (1.8) that

$$
\begin{array}{lll}
|b(a s)| \geqslant a^{\frac{1}{v}}|b(s)|, & |\beta(a s)| \leqslant a^{\nu}|\beta(s)|, & a>1, s \in \mathbb{R}, \\
|b(\alpha s)| \leqslant \alpha^{\frac{1}{v}}|b(s)|, & |\beta(\alpha s)| \geqslant \alpha^{\nu}|\beta(s)|, & 0<\alpha<1, s \in \mathbb{R} .
\end{array}
$$

Therefore, recalling the definition of $\varphi$,

$$
\varphi(a s) \geqslant a^{\frac{1}{v}+1} \varphi(s), \quad \varphi^{-1}(a s) \leqslant a^{\frac{v}{v+1}} \varphi^{-1}(s), \quad a>1, s \in \mathbb{R} .
$$

For all $s \in \mathbb{R}$ we have

$$
|s|^{p} \leqslant \mathcal{D}(s)^{p-1} \varphi(s),
$$

as one can check simply applying Hölder's inequality to

$$
s=\int_{0}^{s} b(\tau)^{1 / p} b(\tau)^{-1 / p} \mathrm{~d} \tau .
$$

The function $\mathcal{D}$ also satisfies for $a>1, s>0$,

$$
\mathcal{D}(a s)=a \int_{0}^{s} b(a \tau)^{-\frac{1}{p-1}} \mathrm{~d} \tau \leqslant a \int_{0}^{s} b(\tau)^{-\frac{1}{p-1}} \mathrm{~d} \tau=a \mathcal{D}(s) .
$$

If we assume (1.10), we get analogously

$$
\begin{array}{lll}
|b(a s)| \leqslant a^{\frac{1}{\mu}}|b(s)|, & |\beta(a s)| \geqslant a^{\mu}|\beta(s)|, & a>1, s \in \mathbb{R}, \\
|b(\alpha s)| \geqslant \alpha^{\frac{1}{\mu}}|b(s)|, & |\beta(\alpha s)| \leqslant \alpha^{\mu}|\beta(s)|, & 0<\alpha<1, s \in \mathbb{R},
\end{array}
$$

and

$$
\varphi(\alpha s) \geqslant \alpha^{\frac{1}{\mu}+1} \varphi(s), \quad \varphi^{-1}(\alpha s) \leqslant \alpha^{\frac{\mu}{\mu+1}} \varphi^{-1}(s), \quad 0<\alpha<1, s \in \mathbb{R} .
$$

Moreover, if (1.10) holds with $\mu(p-1)>1$, one has for $0<\alpha<1, s>0$,

$$
\mathcal{D}(\alpha s)=\alpha \int_{0}^{s} b(\alpha \tau)^{-\frac{1}{p-1}} \mathrm{~d} \tau \leqslant \alpha^{\tilde{\lambda}} \int_{0}^{s} b(\tau)^{-\frac{1}{p-1}} \mathrm{~d} \tau=\alpha^{\tilde{\lambda}} \mathcal{D}(s),
$$

where we exploited (3.6), and set $\tilde{\lambda}=1-1 /(\mu(p-1))>0$. 
Lemma 3.1 Assume (1.7), (1.8), (1.11), and let

$$
u \in W_{\varphi, \mathrm{loc}}^{m, p}\left(\mathbb{R}^{N}\right)=\left\{f \in W_{\mathrm{loc}}^{m, p}\left(\mathbb{R}^{N}\right) \mid \varphi(f) \in L_{\mathrm{loc}}^{1}\left(\mathbb{R}^{N}\right)\right\} .
$$

Then for all $\rho>0,1>\varepsilon>0$,

$$
\int_{G_{\rho}}|u|^{p} \mathrm{~d} x \leqslant \varepsilon \rho^{m p} \int_{G_{\rho}}\left|D^{m} u\right|^{p} \mathrm{~d} x+\gamma \varepsilon^{-\omega} \mathcal{H}_{\rho}[u],
$$

where

$$
\mathcal{H}_{\rho}[u]=\int_{G_{\rho}} \varphi(u) \mathrm{d} x \mathcal{D}\left(\varphi^{-1}\left[\rho^{-N} \int_{G_{\rho}} \varphi(u) \mathrm{d} x\right]\right)^{p-1}
$$

and $\omega=N v(p-1) /(p v+p)$. Here the domain $G_{\rho}$ is equal either to $B_{\rho}=\{|x|<\rho\}$, or to $\Gamma_{\rho}=\left\{\alpha_{1} \rho<|x|<\alpha_{2} \rho\right\}$, where $0<\alpha_{1}<\alpha_{2}$. In this last case, $\gamma$ depends also on $\alpha_{1}, \alpha_{2}, \alpha_{2}-\alpha_{1}$. We note again that $\gamma$ in (3.10) is independent of $\varepsilon$ and of $\rho$.

Proof. Define for $\rho, k>0$,

$$
A_{\rho}(k)=\left\{x \in G_{\rho}|| u \mid>k\right\} .
$$

Then, obviously

$$
\int_{A_{\rho}(k)}|u|^{p} \mathrm{~d} x \leqslant 2^{p-1}\left\{\int_{A_{\rho}(k)}[|u|-k]^{p} \mathrm{~d} x+k^{p}\left|A_{\rho}(k)\right|\right\} .
$$

Moreover, from a well known integral inequality (see, e.g. [29] Chapter II, Lemma 5.1)

$$
\int_{A_{\rho}(k)}[|u|-k]^{p} \mathrm{~d} x \leqslant \gamma_{1}\left|A_{\rho}(k)\right|^{\frac{p}{N}} \int_{A_{\rho}(k)}|D u|^{p} \mathrm{~d} x,
$$

provided

$$
\left|A_{\rho}(k)\right| \leqslant \delta \rho^{N}, \quad 0<\delta<\delta_{0},
$$

where $\delta_{0}$ depends on $N$, and on $\alpha_{1}, \alpha_{2}$ too if $G_{\rho}=\Gamma_{\rho}$. In this connection, we should remark that inequalities like (3.12) are, in general, in force in convex domains. Nevertheless, we are able to prove it even in the annulus $\Gamma_{\rho}$ owing to assumptions (3.13). Essentially, the proof is the same as in [29], when we exploit the remark that

$$
\left|\Gamma_{\rho}(x)-A_{\rho}(k)\right| \geqslant \gamma_{0} \rho^{N}, \quad \text { for all } x \in \Gamma_{\rho},
$$

where $\Gamma_{\rho}(x)=\left\{y \in \Gamma_{\rho} \mid t y+(1-t) x \in \Gamma_{\rho}, \forall 0<t<1\right\}$, if $\delta_{0}$ is suitably chosen.

Moreover, Chebichev's inequality yields, together with (1.11), and for the same constant $c_{1}$ introduced there,

$$
\left|A_{\rho}(k)\right| \leqslant \frac{c_{1}}{\varphi(k)} \int_{G_{\rho}} \varphi(u) \mathrm{d} x .
$$


Recalling (3.4) we get

$$
k^{p}\left|A_{\rho}(k)\right| \leqslant c_{1} \mathcal{D}(k)^{p-1} \int_{G_{\rho}} \varphi(u) \mathrm{d} x .
$$

Note also that, by the same token,

$$
\int_{G_{\rho}-A_{\rho}(k)}|u|^{p} \mathrm{~d} x \leqslant \int_{G_{\rho}-A_{\rho}(k)} \mathcal{D}(u)^{p-1} \varphi(u) \mathrm{d} x \leqslant c_{1} \mathcal{D}(k)^{p-1} \int_{G_{\rho}} \varphi(u) \mathrm{d} x .
$$

Thus, putting (3.11)-(3.16) together, we infer

$$
\int_{G_{\rho}}|u|^{p} \mathrm{~d} x \leqslant \gamma_{2}\left\{\left|A_{\rho}(k)\right|^{\frac{p}{N}} \int_{A_{\rho}(k)}|D u|^{p} \mathrm{~d} x+\mathcal{D}(k)^{p-1} \int_{G_{\rho}} \varphi(u) \mathrm{d} x\right\},
$$

where we have to assume (3.13). Next, for a given $1>\tilde{\varepsilon}>0$, we select $\delta<\delta_{0}$ so that

$$
\gamma_{2} \delta^{\frac{p}{N}} \leqslant \tilde{\varepsilon}
$$

Then we choose $k$ from

$$
\frac{c_{1}}{\varphi(k)} \int_{G_{\rho}} \varphi(u) \mathrm{d} x=\delta \rho^{N},
$$

so that, by virtue of (3.14), (3.13) certainly holds. Therefore, (3.17) gives

$$
\frac{1}{\rho^{p}} \int_{G_{\rho}}|u|^{p} \mathrm{~d} x \leqslant \tilde{\varepsilon} \int_{G_{\rho}}|D u|^{p} \mathrm{~d} x+C_{\tilde{\varepsilon}} \frac{1}{\rho^{p}} \mathcal{H}_{\rho}[u],
$$

where, taking into account (3.3) and (3.5),

$$
C_{\tilde{\varepsilon}} \leqslant \gamma \tilde{\varepsilon}^{-\omega}, \quad \omega=N v(p-1) /(v p+p) .
$$

Finally, the classical John-Nirenberg-Gagliardo inequality, together with Young's inequality, imply

$$
\begin{aligned}
\int_{G_{\rho}}|D u|^{p} \mathrm{~d} x & \leqslant \gamma_{3}\left\{\left(\int_{G_{\rho}}\left|D^{m} u\right|^{p} \mathrm{~d} x\right)^{\frac{1}{m}}\left(\int_{G_{\rho}}|u|^{p} \mathrm{~d} x\right)^{\frac{m-1}{m}}+\frac{1}{\rho^{p}} \int_{G_{\rho}}|u|^{p} \mathrm{~d} x\right\} \\
& \leqslant \frac{\gamma_{4}}{\rho^{p}} \int_{G_{\rho}}|u|^{p} \mathrm{~d} x+\rho^{p(m-1)} \int_{G_{\rho}}\left|D^{m} u\right|^{p} \mathrm{~d} x .
\end{aligned}
$$

If we select $\tilde{\varepsilon} \in\left(0,1 /\left(2 \gamma_{3}\right)\right]$, (3.10) immediately follows for $\varepsilon=2 \tilde{\varepsilon}$.

To prove (3.10) even for $\varepsilon \in\left(1 / \gamma_{3}, 1\right)$, we choose $\tilde{\varepsilon}=1 /\left(2 \gamma_{3}\right)$ in (3.10). Next we take into account that, with this choice of $\tilde{\varepsilon}$, we have $2 \tilde{\varepsilon}<\varepsilon<1$, so that the first term on the right-hand side of (3.10) (written for $\tilde{\varepsilon}$ ) can be bound above by replacing $2 \tilde{\varepsilon}$ with $\varepsilon$, and the second term can be majorized simply by multiplying it by $\varepsilon^{-\omega}>1$.

REMARK 3.1 When $b$ is a power function, say $\beta(s)=s^{\alpha}, \alpha>1 /(p-1)$, the quantity $\mathcal{H}_{\rho}[u]$ can be written more explicitly as

$$
\mathcal{H}_{\rho}[u]=\left(\frac{\alpha(p-1)}{\alpha(p-1)-1}\right)^{p-1} \frac{\alpha}{\alpha+1} \rho^{-\frac{N \alpha(p-1)-N}{\alpha+1}}\|u\|_{\frac{\alpha+1}{\alpha}, G_{\rho}}^{p} .
$$

Note that $\frac{\alpha+1}{\alpha}<p$ if and only if $\alpha(p-1)>1$. 
The following result on cutoff functions is proven essentially as in [28], though in a different geometry; we give here the proof to establish the needed dependence of the estimate on the geometry of the domain.

LemMA 3.2 Let $\rho>0$ and $\alpha_{2} \geqslant \alpha_{1}+1 / 2,0<\alpha<\alpha_{1}$ be given. Define

$$
S_{\rho}=\left\{x \in \mathbb{R}^{N}\left|\left(\alpha_{1}-\alpha\right) \rho<\right| x \mid<\left(\alpha_{2}+\alpha\right) \rho\right\} .
$$

Then there exists a Lipschitz-continuous function $\zeta \geqslant 0$, such that its support is $\bar{S}_{\rho}, \zeta(x) \equiv 1$ in $\left\{\alpha_{1} \rho<|x|<\alpha_{2} \rho\right\}, \zeta \in C^{\infty}\left(S_{\rho}\right)$, and $\left|D^{k} \zeta\right| \leqslant \gamma(N, k) \alpha^{-k} \rho^{-k}$ in $S_{\rho}$. Moreover, for $\ell \geqslant m$, $0<k \leqslant m, 0<\varepsilon<1, u \in W^{m, p}\left(S_{\rho}\right)$, we have

$$
(\alpha \rho)^{-k}\left\|\zeta^{\ell-k} D^{m-k} u\right\|_{p, S_{\rho}} \leqslant \varepsilon\left\|\zeta^{\ell} D^{m} u\right\|_{p, S_{\rho}}+\gamma \varepsilon^{-\frac{m-k}{k}}(\alpha \rho)^{-m}\left\|\zeta^{\ell-m} u\right\|_{p, S_{\rho}} .
$$

Proof. Let $\psi:[0,+\infty) \rightarrow[0,+\infty)$ be a function such that $\psi(s)=1$ for $\alpha_{1}<s<\alpha_{2}$, and

$$
\begin{array}{ll}
\psi(s)=\alpha^{-1}\left(s-\alpha_{1}-\alpha\right)_{+}, & s \leqslant \alpha_{1}-\alpha / 2, \\
\psi(s)=\alpha^{-1}\left(\alpha_{2}+\alpha-s\right)_{+}, & s \geqslant \alpha_{2}+\alpha / 2 .
\end{array}
$$

We also require $\psi \in C^{\infty}\left(\left[\alpha_{1}-\alpha / 2, \alpha_{2}+\alpha / 2\right]\right)$. Of course we may assume $\left|\psi^{(k)}(s)\right| \leqslant \gamma \alpha^{-k}$, $k \geqslant 1$ for $\alpha_{1}-\alpha / 2 \leqslant s \leqslant \alpha_{2}+\alpha / 2$. Then, define $\zeta(x)=\psi(|x| / \rho)$ and

$$
r_{i}^{\prime}=\rho\left(\alpha_{1}-\alpha+\frac{\alpha}{2^{i}}\right), \quad r_{i}^{\prime \prime}=\rho\left(\alpha_{2}+\alpha-\frac{\alpha}{2^{i}}\right), \quad i \geqslant 1 .
$$

Then we have

$$
2^{-i-1} \leqslant \zeta(x) \leqslant 2^{-i} \quad \text { in } \quad \Sigma_{i}=\left\{r_{i+1}^{\prime}<|x|<r_{i}^{\prime}\right\} \cup\left\{r_{i}^{\prime \prime}<|x|<r_{i+1}^{\prime \prime}\right\} .
$$

Applying the John-Gagliardo-Nirenberg inequality in $\Sigma_{i}$ we get for $0<k \leqslant m, 0<\varepsilon<1$,

$$
\left(\alpha 2^{-i} \rho\right)^{-k p}\left\|D^{m-k} u\right\|_{p, \Sigma_{i}}^{p} \leqslant \varepsilon^{p}\left\|D^{m} u\right\|_{p, \Sigma_{i}}^{p}+\gamma \varepsilon^{-\frac{m-k}{k} p}\left(\alpha 2^{-i} \rho\right)^{-m p}\|u\|_{p, \Sigma_{i}}^{p} .
$$

Next we multiply (3.22) by $2^{-i \ell p}$ and, invoking (3.21), we arrive at

$$
(\alpha \rho)^{-k p}\left\|\zeta^{\ell-k} D^{m-k} u\right\|_{p, \Sigma_{i}}^{p} \leqslant \varepsilon^{p}\left\|\zeta^{\ell} D^{m} u\right\|_{p, \Sigma_{i}}^{p}+\gamma \varepsilon^{-\frac{m-k}{k} p}(\alpha \rho)^{-m p}\left\|\zeta^{\ell-m} u\right\|_{p, \Sigma_{i}}^{p} .
$$

Note that a similar inequality holds for the domain $S^{\prime}=\left\{\alpha_{1}-\alpha / 2<|x|<\alpha_{2}+\alpha / 2\right\}$ : indeed we can introduce the scaling factor $\alpha<1$ in this inequality by a suitable choice of $\varepsilon$. Finally, we get (3.20) by summing the inequalities (3.23), over $i=1,2, \ldots$ and then adding to the resulting inequality the quoted estimate valid in $S^{\prime}$.

\section{Proof of Theorem 1.1}

For $\rho>4 r_{0}$, define for $\frac{1}{4}>\theta_{2}>\theta_{1}>0$, and $i=0,1, \ldots$

$$
r_{i}^{\prime}=\frac{\rho}{2}-\theta_{2} \rho+\frac{\rho}{2^{i}}\left(\theta_{2}-\theta_{1}\right), \quad r_{i}^{\prime \prime}=\rho+\theta_{2} \rho-\frac{\rho}{2^{i}}\left(\theta_{2}-\theta_{1}\right) .
$$


Define also the sequence of annuli $A_{i}=\left\{r_{i}^{\prime}<|x|<r_{i}^{\prime \prime}\right\} \subset A_{i+1}$. Let $\zeta_{i}, i \geqslant 1$, be the function constructed in Lemma 3.2, where we define $\theta=\theta_{2}-\theta_{1}$ and

$$
\alpha_{1}=\frac{1}{2}-\theta_{2}+\frac{\theta}{2^{i}}, \quad \alpha=\frac{\theta}{2^{i+1}}, \quad \alpha_{2}=1+\theta_{2}-\frac{\theta}{2^{i}} .
$$

Then $\zeta_{i} \equiv 1$ in $A_{i}, \zeta \equiv 0$ out of $A_{i+1}$, and $\left|D^{k} \zeta_{i}\right| \leqslant \gamma\left(2^{-i} \rho \theta\right)^{-k}$. Next we formally take $\zeta_{i}^{s} u$ as a testing function in the weak formulation of (1.5), (1.6), where $s>m$ is to be chosen large enough. More exactly, we invoke (1.15) in definition 1.1, to obtain

$$
\begin{aligned}
\sup _{0 \leqslant \tau \leqslant t} \int_{\mathbb{R}^{N}} \Phi(u) \zeta_{i}^{s} \mathrm{~d} x+\int_{0}^{t} \int_{\mathbb{R}^{N}} \zeta_{i}^{s}\left|D^{m} u\right|^{p} \mathrm{~d} x \mathrm{~d} \tau & \\
& \leqslant \gamma \sum_{k=1}^{m}\left(2^{-i} \theta \rho\right)^{-k} \int_{0}^{t} \int_{\mathbb{R}^{N}} \zeta_{i}^{s-k}\left|D^{m-k} u\right|\left|D^{m} u\right|^{p-1} \mathrm{~d} x \mathrm{~d} \tau
\end{aligned}
$$

where

$$
\Phi(u)=\int_{0}^{b(u)} \beta(z) \mathrm{d} z, \quad u \in \mathbb{R} .
$$

Next, for $\varepsilon \in(0,1)$, we majorize the right-hand side of (4.1) by means of Young's inequality, obtaining

$$
\varepsilon \int_{0}^{t} \int_{\mathbb{R}^{N}}\left|D^{m} u\right|^{p} \zeta_{i}^{s} \mathrm{~d} x \mathrm{~d} \tau+\gamma \varepsilon^{-p+1} \sum_{k=1}^{m}\left(2^{-i} \theta \rho\right)^{-k p} \int_{0}^{t} \int_{\mathbb{R}^{N}}\left|D^{m-k} u\right|^{p} \zeta_{i}^{s-k p} \mathrm{~d} x \mathrm{~d} \tau
$$

In turn, setting $s=\ell p, \ell>m$, we bound above the second term in (4.2): exploiting Lemma 3.2 we get the bound

$$
\gamma \varepsilon^{-p+1} \tilde{\varepsilon} \int_{0}^{t} \int_{\mathbb{R}^{N}} \zeta_{i}^{\ell p}\left|D^{m} u\right|^{p} \mathrm{~d} x \mathrm{~d} \tau+\gamma \varepsilon^{-p+1} \tilde{\varepsilon}^{1-m}\left(2^{-i} \theta \rho\right)^{-m p} \int_{0}^{t} \int_{\mathbb{R}^{N}} \zeta_{i}^{(\ell-m) p}|u|^{p} \mathrm{~d} x \mathrm{~d} \tau
$$

where we choose $\varepsilon$ and then $\tilde{\varepsilon}$ so that we can absorb the terms containing $\left|D^{m} u\right|$ and appearing in (4.2), (4.3) into the left-hand side of (4.1).

Note that, integrating by parts and applying (1.8), one gets

$$
\Phi(u) \geqslant(1+v)^{-1} \varphi(u), \quad u \in \mathbb{R} .
$$

Therefore, we arrive at

$$
\sup _{0 \leqslant \tau \leqslant t} \int_{\mathbb{R}^{N}} \varphi(u) \zeta_{i}^{\ell p} \mathrm{~d} x+\int_{0}^{t} \int_{\mathbb{R}^{N}} \zeta_{i}^{\ell p}\left|D^{m} u\right|^{p} \mathrm{~d} x \mathrm{~d} \tau \leqslant \gamma\left(2^{-i} \theta \rho\right)^{-m p} \int_{0}^{t} \int_{A_{i+1}}|u|^{p} \mathrm{~d} x \mathrm{~d} \tau .
$$

Next, we invoke Lemma 3.1 to majorize the right-hand side of (4.5) with

$$
\varepsilon \int_{0}^{t} \int_{A_{i+1}}\left|D^{m} u\right|^{p} \mathrm{~d} x \mathrm{~d} \tau+\gamma \varepsilon^{-\omega} \frac{t}{\rho^{m p}}\left(2^{-i} \theta\right)^{-q p} \mathcal{H}_{\rho}^{\infty}[u]
$$


where $q=(\omega+1) m, \omega$ as in Lemma 3.1, and we set

$$
\mathcal{H}_{\rho}^{\infty}[u]=\sup _{0 \leqslant \tau \leqslant t} \int_{A_{\infty}} \varphi(u(x, \tau)) \mathrm{d} x \mathcal{D}\left(\varphi^{-1}\left[\rho^{-N} \sup _{0 \leqslant \tau \leqslant t} \int_{A_{\infty}} \varphi(u(x, \tau)) \mathrm{d} x\right]\right)^{p-1},
$$

with $A_{\infty}=\left\{\rho\left(2^{-1}-\theta_{2}\right)<|x|<\rho\left(1+\theta_{2}\right)\right\}$. Choosing $\varepsilon>0$ small enough (in fact, $\varepsilon<2^{-q p}$ ), we obtain, after an easy iteration procedure (see, e.g. [22]),

$$
\sup _{0 \leqslant \tau \leqslant t} \int_{A_{0}} \varphi(u) \mathrm{d} x+\int_{0}^{t} \int_{A_{0}}\left|D^{m} u\right|^{p} \mathrm{~d} x \mathrm{~d} \tau \leqslant \gamma \theta^{-q p} \frac{t}{\rho^{m p}} \mathcal{H}_{\rho}^{\infty}[u] .
$$

We conclude the proof by applying again an iterative technique: let $\rho>4 r_{0}$ and

$$
\rho_{n}^{\prime}=\frac{\rho}{2}-\sigma \frac{\rho}{2^{n}}, \quad \rho_{n}^{\prime \prime}=\rho+\sigma \frac{\rho}{2^{n}}, \quad n=0,1, \ldots
$$

We choose in the first part of the proof

$$
\theta_{2}=\sigma 2^{-n}, \quad \theta_{1}=\sigma 2^{-n-1}, \quad \theta=\sigma 2^{-n-1},
$$

with $\sigma \in(0,1 / 4)$. Defining

$$
Y_{n}=\sup _{0 \leqslant \tau \leqslant t} \frac{1}{\rho^{N}} \int_{\left\{\rho_{n}^{\prime}<|x|<\rho_{n}^{\prime \prime}\right\}} \varphi(u(x, \tau)) \mathrm{d} x,
$$

it follows from (4.7) that for all $n \geqslant 0$,

$$
Y_{n+1} \leqslant \gamma(\sigma) \frac{t}{\rho^{m p}} 2^{n q p} Y_{n} \mathcal{D}\left(\varphi^{-1}\left(Y_{n}\right)\right)^{p-1} .
$$

To prove (1.20), we observe that, if (1.10) holds, we may invoke (3.8), with $\alpha=Y_{n} / Y_{0}$, to get from (4.9)

$$
Y_{n+1} \leqslant \gamma \frac{t}{\rho^{m p}} 2^{n q p} Y_{n}^{1+\Lambda}\left[Y_{0}^{-\Lambda} \mathcal{D}\left(\varphi^{-1}\left(Y_{0}\right)\right)^{p-1}\right],
$$

where $\Lambda=\lambda \mu(p-1) /(\mu+1)$, and we have made use also of assumption (1.9). Thus, Lemma 5.6 in Chapter II of [29] implies that $Y_{n} \rightarrow 0$ if $\rho$ is so large that

$$
Y_{0}^{\Lambda} \frac{t}{\rho^{m p}}\left[Y_{0}^{-\Lambda} \mathcal{D}\left(\varphi^{-1}\left(Y_{0}\right)\right)^{p-1}\right] \leqslant \gamma_{0} .
$$

Therefore, recalling the definition of $Y_{n}$, we conclude that, under this assumption, $u(x, t) \equiv 0$ for $|x|>\rho / 2$. Using now the bound $Y_{n} \leqslant \rho^{-N}\left\|\varphi\left(u_{0}\right)\right\|_{1, \mathbb{R}^{N}}$, see remark 1.3, we finally infer (1.20), after an elementary procedure of functional inversion.

Let us now turn to the proof of (1.19). Even if (1.10) does not hold, we may invoke (3.4) to write, for $s \leqslant Y_{0}$,

$$
\varphi^{-1}(s) \leqslant \mathcal{D}\left(\varphi^{-1}(s)\right)^{\frac{p-1}{p}} s^{\frac{1}{p}} \leqslant \mathcal{D}\left(\varphi^{-1}\left(Y_{0}\right)\right)^{\frac{p-1}{p}} s^{\frac{1}{p}}
$$


Let $d_{0}=\mathcal{D}\left(\varphi^{-1}\left(Y_{0}\right)\right)^{\frac{p-1}{p}}, z_{n}=Y_{n}^{\frac{1}{p}}$. Then (4.9) gives

$$
z_{n+1} \leqslant \gamma\left(\frac{t}{\rho^{m p}}\right)^{\frac{1}{p}} 2^{n q} z_{n} \mathcal{D}\left(d_{0} z_{n}\right)^{\frac{p-1}{p}} \leqslant \gamma\left(\frac{t}{\rho^{m p}}\right)^{\frac{1}{p}} 2^{n q} z_{n}^{1+\frac{p-1}{p} \lambda}\left[z_{0}^{-\lambda} \mathcal{D}\left(d_{0} z_{0}\right)\right]^{\frac{p-1}{p}}
$$

again by means of (1.9). As above, we have that $z_{n} \rightarrow 0$ if

$$
\left(\frac{t}{\rho^{m p}}\right)^{\frac{1}{p}} z_{0}^{\frac{p-1}{p} \lambda}\left[z_{0}^{-\lambda} \mathcal{D}\left(d_{0} z_{0}\right)\right]^{\frac{p-1}{p}} \leqslant \gamma_{0}
$$

yielding finally the estimate (1.19). Indeed, we see that $d_{0}$ and $z_{0}$ can be bounded in terms of $\left\|\Phi\left(u_{0}\right)\right\|_{1, \mathbb{R}^{N}}$, when we note that, by the first inequality in (1.17), and by (1.16), we have

$$
Y_{0} \leqslant \sup _{\tau>0} \int_{\mathbb{R}^{N}} \varphi(u(x, \tau)) \mathrm{d} x \leqslant(1+v) \sup _{\tau>0} \int_{\mathbb{R}^{N}} \Phi(u(x, \tau)) \mathrm{d} x \leqslant(1+v)\left\|\Phi\left(u_{0}\right)\right\|_{1, \mathbb{R}^{N}} .
$$

The proof of Theorem 1.1 is concluded.

REMARK 4.1 The proof above makes use of the partial differential equation only to derive the energy inequality (4.1). In the case of (1.26) this formula is changed only because the term $\left|D^{m} u\right|$ is replaced by $\left|\Delta^{\frac{m}{2}} u\right|$ both on the left-hand side and on the right-hand side. On applying Young's inequality as in (4.2), we arrive at

$$
\begin{aligned}
\sup _{0 \leqslant \tau \leqslant t} \int_{\mathbb{R}^{N}} \Phi(u) \zeta_{i}^{s} \mathrm{~d} x+\int_{0}^{t} \int_{\mathbb{R}^{N}} \zeta_{i}^{s}\left|\Delta^{\frac{m}{2}} u\right|^{p} \mathrm{~d} x \mathrm{~d} \tau & \\
& \leqslant \gamma \sum_{k=1}^{m}\left(2^{-i} \theta \rho\right)^{-k p} \int_{0}^{t} \int_{\mathbb{R}^{N}} \zeta_{i}^{s-k p}\left|D^{m-k} u\right|^{p} \mathrm{~d} x \mathrm{~d} \tau .
\end{aligned}
$$

Clearly, we may replace the integrand in the second integral on the left-hand side with $\left|\Delta^{\frac{m}{2}}\left(u \zeta_{i}^{s / p}\right)\right|$, at the only cost of changing the constant $\gamma$ appearing on the right-hand side. Next, we recall Calderon-Zygmund inequality (see, e.g. [34] p.59): $\left\|D^{m} v\right\|_{p, \mathbb{R}^{N}} \leqslant \gamma\left\|\Delta^{\frac{m}{2}} v\right\|_{p, \mathbb{R}^{N}}$, which is valid for compactly supported $v$, with $\gamma$ depending on $p, N, m$. We apply it to $v=u \zeta_{i}^{s / p}$. This allows us to bound below the left-hand side of (4.11) by formally substituting $\zeta_{i}^{s}\left|\Delta^{\frac{m}{2}} u\right|^{p}$ with $\left|D^{m}\left(u \zeta_{i}^{s / p}\right)\right|^{p}$ there. The proof can then be continued as above.

\section{Proof of Theorem 1.2}

We may assume $u \geqslant 0$, by working separately with the positive and the negative part of $u$.

Define the sequences

$$
t_{n}=\left(1-\sigma 2^{-n}\right) t / 2, \quad k_{n}=\left(1-2^{-n-1}\right) k, \quad n \geqslant 0,
$$

where $t>0$ and $\sigma \in\left(0, \frac{1}{2}\right)$ are arbitrarily fixed, while $k>0$ is to be chosen. Let $Q_{n}=\mathbb{R}^{N} \times\left(t_{n}, t\right)$, and let $\zeta_{n}=\zeta_{n}(t)$ be a smooth function, $0 \leqslant \zeta_{n} \leqslant 1, \zeta_{n} \equiv 1$ for $t \geqslant t_{n+1}, \zeta_{n} \equiv 0$ for $t \leqslant t_{n}$, $0 \leqslant \zeta_{n t} \leqslant \gamma 2^{n} /(\sigma t)$. 
The proof is in fact based on an iteration procedure that is very similar to the ones in $[2,3]$, so we confine ourselves to a sketch of the main differences. In the proof of the sup estimate (1.21) we work with the formulation of (1.5), (1.6) where the unknown is $v=b(u)$. In the corresponding weak formulation we select $\eta \equiv \zeta_{n+1}^{p}\left[\beta(v)-\beta\left(k_{n+1}\right)\right]_{+}$as a testing function. From the definition of solution it follows that

$$
\begin{aligned}
& \int_{\mathbb{R}^{N}(t)} F\left(v, k_{n+1}\right)^{2} \zeta_{n+1}^{p} \mathrm{~d} x+\int_{0}^{t} \int_{\mathbb{R}^{N}} D \eta \cdot D \beta(v)|D \beta(v)|^{p-2} \mathrm{~d} x \mathrm{~d} \tau \\
&=\int_{0}^{t} \int_{\mathbb{R}^{N}} F\left(v, k_{n+1}\right)^{2} \frac{\partial}{\partial \tau}\left(\zeta_{n+1}^{p}\right) \mathrm{d} x \mathrm{~d} \tau
\end{aligned}
$$

where

$$
F(v, k)=\left[\int_{k}^{v}(\beta(s)-\beta(k))_{+} \mathrm{d} s\right]^{\frac{1}{2}} .
$$

Routine calculations lead us to

$$
\begin{aligned}
\sup _{0 \leqslant \tau \leqslant t} \int_{\mathbb{R}^{N}} F\left(v, k_{n+1}\right)^{2} \zeta_{n+1}^{p} \mathrm{~d} x+\int_{0}^{t} \int_{v>k_{n+1}}|D \beta(v)|^{p} \zeta_{n+1}^{p} \mathrm{~d} x \mathrm{~d} \tau & \\
& \leqslant \gamma \frac{2^{n}}{\sigma t} \iint_{Q_{n}} F\left(v, k_{n}\right)^{2} \mathrm{~d} x \mathrm{~d} \tau .
\end{aligned}
$$

Note that

$$
D F\left(v, k_{n}\right)=D v\left[\beta(v)-\beta\left(k_{n}\right)\right]_{+}\left(2 F\left(v, k_{n}\right)\right)^{-1},
$$

whence

$$
\left|D F\left(v, k_{n+1}\right)\right| \leqslant \gamma|D v|\left(\frac{\beta(v)}{v}\right)^{\frac{1}{2}} \chi_{\left\{v>k_{n+1}\right\}} .
$$

This can be shown, in the case $v>C k, C=2^{1+v / \mu}$, by combining (5.3) with the following estimate:

$$
\begin{aligned}
\frac{\beta(v)}{v} \int_{k_{n+1}}^{v}\left(\beta(s)-\beta\left(k_{n+1}\right)\right)_{+} \mathrm{d} s & \geqslant \frac{\beta(v)}{v} \int_{\frac{v}{2}}^{v}\left(\beta(s)-\beta\left(\frac{v}{C}\right)\right)_{+} \mathrm{d} s \\
& \geqslant \frac{\beta(v)}{v}\left[\beta\left(\frac{v}{2}\right)-\beta\left(\frac{v}{C}\right)\right] \frac{v}{2} \geqslant \frac{\beta(v)^{2}}{2}\left[\frac{1}{2^{v}}-\frac{1}{C^{\mu}}\right] \geqslant \gamma_{0} \beta(v)^{2} .
\end{aligned}
$$

The case $v \leqslant C k$ follows even more simply. Therefore, from (5.2) we arrive at

$$
\begin{array}{r}
\sup _{0 \leqslant \tau \leqslant t} \int_{\mathbb{R}^{N}} F\left(v, k_{n+1}\right)^{2} \zeta_{n+1}^{p} \mathrm{~d} x+\left[\frac{\beta(k)}{k}\right]^{\frac{p}{2}} \int_{0}^{t} \int_{\mathbb{R}^{N}}\left|D F\left(v, k_{n+1}\right)\right|^{p} \zeta_{n+1}^{p} \mathrm{~d} x \mathrm{~d} \tau \\
\leqslant \gamma \frac{2^{n}}{\sigma t} \iint_{Q_{n}} F\left(v, k_{n}\right)^{2} \mathrm{~d} x \mathrm{~d} \tau
\end{array}
$$


where we used $\mu \geqslant 1$ to ensure that $\beta(s) / s$ is nondecreasing in $s$. Then the proof of (1.21) can be concluded as in the papers quoted above.

In order to prove (1.22) we need to multiply the equation by $u^{\theta}$, for a small $\theta>0$. This leads us to consider for any fixed $\theta \in(0,1)$ the function

$$
\xi(s)=s^{\frac{p}{p+\theta-1}(\theta-1)} \varphi\left(s^{\frac{p}{p+\theta-1}}\right), \quad s>0 .
$$

As an easy consequence of assumptions (1.8) and (1.10), $\xi$ is increasing and

$$
\begin{array}{ll}
\xi^{-1}(a s) \leqslant a^{c} \xi^{-1}(s), & a>1, s>0, \\
\xi^{-1}(\alpha s) \leqslant \alpha^{d} \xi^{-1}(s), & 0<\alpha<1, s>0,
\end{array}
$$

where $c=c(\theta, p, v)$ and $d=\mu(p+\theta-1) / p(\theta \mu+1)$. Let us also introduce the integral

$$
I_{\rho}=\frac{1}{\rho^{N}} \int_{G_{\rho}} \xi(u(x)) \mathrm{d} x, \quad G_{\rho} \subset \mathbb{R}^{N},
$$

and the function

$$
\mathcal{K}(s)=\xi^{-1}(s)^{1-\theta} \mathcal{D}\left(\xi^{-1}(s)^{\frac{p}{p+\theta-1}}\right)^{\frac{p+\theta-1}{p}(p-1)} s^{\frac{p+\theta-1}{p}}, \quad s>0,
$$

arising naturally in Lemma 5.2 below.

Lemma 5.1 Let $0 \leqslant u \in W^{1, p}\left(G_{\rho}\right), \theta \in(0,1)$, and assume (1.8), (1.7). Then for all $\varepsilon \in(0,1)$

$$
\int_{G_{\rho}} u^{p} \mathrm{~d} x \leqslant \varepsilon \rho^{p} \int_{G_{\rho}}|D u|^{p} \mathrm{~d} x+\gamma \varepsilon^{-h} \rho^{N} \mathcal{K}\left(I_{\rho}\right),
$$

where $G_{\rho}$ is chosen as in Lemma 3.1, and $h=h(N, \theta, p, v)>0$.

Proof. The proof is very close to the one of Lemma 3.1, so that we sketch only the main steps. Let

$$
A_{\rho}(k)=\left\{x \in G_{\rho} \mid u(x)>k\right\}, \quad k>0 .
$$

We can repeat without change the reasoning in (3.11)-(3.13). Again, we apply Chebichev's inequality to prove

$$
\left|A_{\rho}(k)\right| \leqslant \frac{1}{\xi(k)} \int_{G_{\rho}} \xi(u) \mathrm{d} x,
$$

and select $k$ so that the right-hand side of (5.10) equals $\delta \rho^{N}$, with $\delta$ suitably small and $\gamma \delta^{p / N} \leqslant \varepsilon$.

Moreover, note that it follows from (3.4), (5.6) that

$$
u^{p} \leqslant u^{1-\theta} \xi(u)^{\frac{p+\theta-1}{p}} \mathcal{D}\left(u^{\frac{p}{p+\theta-1}}\right)^{\frac{p+\theta-1}{p}(p-1)} .
$$

Therefore, using (5.11) to estimate $u$ in $G_{\rho}-A_{\rho}(k)$, we find, after an application of Hölder's inequality

$$
\int_{G_{\rho}} u^{p} \mathrm{~d} x \leqslant \varepsilon \rho^{p} \int_{G_{\rho}}|D u|^{p} \mathrm{~d} x+\gamma \rho^{N \frac{1-\theta}{p}} k^{1-\theta} \mathcal{D}\left(k^{\frac{p}{p+\theta-1}}\right)^{\frac{p+\theta-1}{p}(p-1)}\left[\int_{G_{\rho}} \xi(u) \mathrm{d} x\right]^{\frac{p+\theta-1}{p}} .
$$

Substituting in the last inequality the definition of $k$, and using (5.7), we get (5.9). 
Lemma 5.2 Let $u \geqslant 0$ be a solution to (1.5), (1.6), with $m=1$. Under the assumptions of Theorem 1.2, we have that $u(x, t)=0$ for $|x| \geqslant \rho$, provided $\rho \geqslant 4 r_{0}$ and

$$
{ }_{t} \rho^{-p} E_{0}^{-1} \mathcal{K}\left(E_{0}\right) \leqslant \gamma_{0},
$$

where $\theta$ in (5.6) is chosen suitably small, and we let

$$
E_{0}=\frac{1}{\rho^{N+p}} \int_{0}^{t} \int_{B_{2 \rho}} u(x, t)^{p+\theta-1} \mathrm{~d} x \mathrm{~d} \tau .
$$

Proof. Define $\rho_{n}^{\prime}, \rho_{n}^{\prime \prime}$ as in (4.8), for $\sigma \in\left(0, \frac{1}{4}\right), \rho>4 r_{0}$. Let $A_{n}=\left\{\rho_{n}^{\prime}<|x|<\rho_{n}^{\prime \prime}\right\}$, and define a sequence of cutoff functions $\zeta_{n}(x)$ so that $\zeta_{n} \equiv 1$ in $A_{n}, \zeta_{n} \equiv 0$ out of $A_{n-1},\left|D \zeta_{n}\right| \leqslant \gamma\left(2^{-n} \sigma \rho\right)^{-1}$. We choose as a test fuction in the weak formulation of the problem the product $u^{\theta} \zeta_{n+1}^{p}$, where $\theta \in(0,1)$ is the same constant appearing in the definition of $\xi$, and will be chosen later. We have, formally,

$$
[b(u)]_{t} u^{\theta}=\frac{\partial}{\partial t} L(u), \quad L(u) \equiv \int_{0}^{b(u)} \beta(s)^{\theta} \mathrm{d} s .
$$

Therefore, integrating by parts we get

$$
\int_{\mathbb{R}^{N}(t)} L(u) \zeta_{n}^{p} \mathrm{~d} x+\int_{0}^{t} \int_{\mathbb{R}^{N}} D\left(L(u) \zeta_{n}^{p}\right) \cdot D u|D u|^{p-2} \mathrm{~d} x \mathrm{~d} \tau=0 .
$$

Moreover, by integrating by parts the definition of $L(u)$, and applying (1.8), we find

$$
\int_{0}^{b(u)} \beta(s)^{\theta} \mathrm{d} s \geqslant \theta(1+\nu \theta)^{-1} \int_{0}^{u} s^{\theta-1} b(s) \mathrm{d} s \geqslant \theta(1+\nu \theta)^{-1} u^{\theta-1} \varphi(u) .
$$

Then, standard calculations yield

$$
\sup _{0 \leqslant \tau \leqslant t} \int_{\mathbb{R}^{N}} \zeta_{n+1}^{p} u^{\theta-1} \varphi(u) \mathrm{d} x+\int_{0}^{t} \int_{\mathbb{R}^{N}} u^{\theta-1}|D u|^{p} \zeta_{n+1}^{p} \mathrm{~d} x \mathrm{~d} \tau \leqslant \gamma \frac{2^{p n}}{\sigma^{p} \rho^{p}} \int_{0}^{t} \int_{A_{n}} u^{p+\theta-1} \mathrm{~d} x \mathrm{~d} \tau .
$$

Defining $\bar{u}=u^{\frac{p+\theta-1}{p}}$, this can be rewritten as

$$
\sup _{0 \leqslant \tau \leqslant t} \int_{\mathbb{R}^{N}} \zeta_{n+1}^{p} \xi(\bar{u}) \mathrm{d} x+\int_{0}^{t} \int_{\mathbb{R}^{N}} \zeta_{n+1}^{p}|D \bar{u}|^{p} \mathrm{~d} x \mathrm{~d} \tau \leqslant \gamma \frac{2^{p n}}{\sigma^{p} \rho^{p}} \int_{0}^{t} \int_{A_{n}} \bar{u}^{p} .
$$

Set

$$
Y_{n}=\sup _{0 \leqslant \tau \leqslant t} \frac{1}{\rho^{N}} \int_{A_{n}} \xi(\bar{u}) \mathrm{d} x .
$$

Then the integral on the right-hand side of (5.14) can be majorized by means of Lemma 5.1, so that

$$
\frac{1}{\rho^{p}} \int_{0}^{t} \int_{A_{n}} \bar{u}^{p} \mathrm{~d} x \mathrm{~d} \tau \leqslant \varepsilon \int_{0}^{t} \int_{A_{n}}|D \bar{u}|^{p} \mathrm{~d} x \mathrm{~d} \tau+\gamma \varepsilon^{-h} t \rho^{N-p} \mathcal{K}\left(Y_{n}\right) .
$$


One can get rid of the first term appearing on the right-hand side of (5.15) by an iteration procedure, exactly as in the proof of Theorem 1.1, provided $\varepsilon$ is chosen small enough. We omit the details, for the sake of brevity. Therefore, we eventually arrive at

$$
Y_{n+1} \leqslant \gamma \gamma_{1}^{n} \frac{t}{\rho^{p}} \mathcal{K}\left(Y_{n}\right), \quad n \geqslant 0 .
$$

Taking into account that $Y_{n} / Y_{0} \leqslant 1$, and by virtue of (5.8), and of (3.9), we see that

$$
\mathcal{K}\left(Y_{n}\right)=\mathcal{K}\left(\frac{Y_{n}}{Y_{0}} Y_{0}\right) \leqslant Y_{n}^{1+\Lambda(\theta)}\left[Y_{0}^{-1-\Lambda(\theta)} \mathcal{K}\left(Y_{0}\right)\right]
$$

where

$$
\Lambda(\theta)=(1-\theta) \frac{\mu(p+\theta-1)}{p(\theta \mu+1)}+\frac{\theta-1}{p}+\left(1-\frac{1}{\mu(p-1)}\right) \frac{\mu(p+\theta-1)}{p(\theta \mu+1)}(p-1) .
$$

Note that $\Lambda(0)=\mu(p-1)-1>0$, so that $\Lambda(\theta)>0$ for small $\theta>0$. Thus we conclude the proof invoking the same result from [29] quoted in the proof of Theorem 1.1, and the fact that $Y_{0}$ can bounded above by $\gamma E_{0}$ by means of calculations similar to the ones leading to (5.14).

In order to complete the proof of (1.22), we have to apply the sup estimate (1.21) in (5.12).

REMARK 5.1 Let us deal first with the case of power functions, both for its intrinsic interest and for its simplicity. Recall that when $\beta(s)=s^{\alpha}, \alpha>1 /(p-1),(1.21)$ takes the form (1.24). Let us apply this estimate in the definition of $E_{0}$ :

$$
\begin{aligned}
E_{0} & =\frac{1}{\rho^{N+p}} \int_{\mathbb{R}^{N}} u^{p+\theta-1} \mathrm{~d} \tau \leqslant \gamma \frac{\left\|v_{0}\right\|_{1, \mathbb{R}^{N}}}{\rho^{N+p}} \int_{0}^{t}\|u(\cdot, \tau)\|_{\infty, \mathbb{R}^{N}}^{p+\theta-1-\frac{1}{\alpha}} \mathrm{d} \tau \\
& \leqslant \gamma \frac{\left\|v_{0}\right\|_{1, \mathbb{R}^{N}}}{\rho^{N+p}} t^{1+\frac{p[\alpha(p-1)-1]+p \alpha \theta}{N \alpha(p-1)-N+p}} t^{\frac{p-N \alpha \theta}{N \alpha(p-1)-N+p}},
\end{aligned}
$$

provided $\theta<p /(N \alpha)$. Note that in the present setting (5.12) can be written as

$$
\frac{t}{\rho^{p}} E_{0}^{\frac{p+\theta-1}{\theta+\frac{1}{\alpha}}-1} \leqslant \gamma_{0} \text {. }
$$

If we substitute the bound (5.16) for $E_{0}$ in this inequality, we discover that (5.12) is fulfilled provided

$$
\rho \geqslant \gamma\left\|v_{0}\right\|_{1, \mathbb{R}^{N}}^{\frac{\alpha(p-1)-1}{N \alpha(p-1)-N+p}} t^{\frac{1}{N \alpha(p-1)-N+p}} .
$$

Recalling Lemma 5.2, we conclude that $Z(t)$ is bounded as in (1.25).

Let us go back to the proof of (1.22) for a general $b$. The sup estimate (1.21) can be obviously rewritten as

$$
\|u(\cdot, t)\|_{\infty, \mathbb{R}^{N}} \leqslant \mathcal{A}_{1}\left(\gamma t^{-\frac{N}{p}}\left\|v_{0}\right\|_{1, \mathbb{R}^{N}}\right)=: \mathcal{A}(t), \quad t>0
$$


where $\mathcal{A}_{1}$ is the inverse function of $s \mapsto b(s) \mathcal{D}(s)^{N(p-1) / p}$. Then, noting that $s \mapsto s^{p+\theta-1} / b(s)$ is increasing owing to $\mu(p-1)>1$, we calculate

$$
E_{0} \leqslant \frac{1}{\rho^{N+p}} \int_{0}^{t} \int_{\mathbb{R}^{N}} u^{p+\theta-1} \mathrm{~d} x \mathrm{~d} \tau \leqslant \gamma \frac{\left\|v_{0}\right\|_{1, \mathbb{R}^{N}}}{\rho^{N+p}} \int_{0}^{t} \frac{\|u(\cdot, \tau)\|_{\infty, \mathbb{R}^{N}}^{p+\theta-1}}{b\left(\|u(\cdot, \tau)\|_{\infty, \mathbb{R}^{N}}\right)} \mathrm{d} \tau .
$$

It follows from (3.4) that $s^{p-1} / b(s) \leqslant \mathcal{D}^{p-1}(s)$. Hence, recalling the definitions of $\mathcal{A}_{1}$ and $\mathcal{A}$, we get for all $\tau \in(0, t)$,

$$
\frac{\|u(\cdot, \tau)\|_{\infty, \mathbb{R}^{N}}^{p+\theta-1}}{b\left(\|u(\cdot, \tau)\|_{\infty, \mathbb{R}^{N}}\right)} \leqslant \mathcal{A}(\tau)^{\theta} \mathcal{D}(\mathcal{A}(\tau))^{p-1} \leqslant \gamma \mathcal{A}(\tau)^{\theta} \frac{\tau^{-1}\left\|v_{0}\right\|_{1, \mathbb{R}^{N}}^{\frac{p}{N}}}{b(\mathcal{A}(\tau))^{\frac{p}{N}}} .
$$

Moreover, again from the definition of $\mathcal{A}(t)$, and from (5.7), (5.8), it follows that

$$
\mathcal{A}(\tau)^{\theta} \frac{\tau^{-1}\left\|v_{0}\right\|_{1, \mathbb{R}^{N}}^{\frac{p}{N}}}{b(\mathcal{A}(\tau))^{\frac{p}{N}}} \leqslant \mathcal{A}(t)^{\theta} \frac{t^{-1}\left\|v_{0}\right\|_{1, \mathbb{R}^{N}}^{\frac{p}{N}}}{b(\mathcal{A}(t))^{\frac{p}{N}}}\left(\frac{t}{\tau}\right)^{\delta}, \quad 0<\tau<t,
$$

for a $\delta \in(0,1)$, provided $\theta$ is chosen small enough. Therefore, using this estimate in (5.17) we draw the conclusion

$$
E_{0} \leqslant \gamma \frac{\left\|v_{0}\right\|_{1, \mathbb{R}^{N}}^{1+\frac{p}{N}}}{\rho^{p+N}} \frac{\mathcal{A}(t)^{\theta}}{b(\mathcal{A}(t))^{\frac{p}{N}}}
$$

Define

$$
\bar{\rho}(t)=C b(\mathcal{A}(t))^{-\frac{1}{N}}\left\|v_{0}\right\|_{1, \mathbb{R}^{N}}^{\frac{1}{N}}, \quad t>0,
$$

where the constant $C>1$ is going to be chosen presently. Just using the definition of $\mathcal{A}(t)$, we see, after elementary calculations, that for $\rho \geqslant \bar{\rho}(t), t>0$,

$$
\frac{t}{\rho^{p}} \leqslant \frac{t}{\bar{\rho}(t)^{p}} \leqslant \frac{\gamma}{C^{p} \mathcal{D}(\mathcal{A}(t))^{p-1}}
$$

Moreover, from (5.18) we find, again for $\rho \geqslant \bar{\rho}(t)$,

$$
E_{0} \leqslant \frac{\gamma}{C^{p+N}} \mathcal{A}(t)^{\theta} b(\mathcal{A}(t)) \leqslant \frac{\gamma}{C^{p+N}} \xi\left(\mathcal{A}(t)^{\frac{p+\theta-1}{p}}\right)
$$

Thus, we get from (5.19), (5.20), and from the definition of $\mathcal{K}$ (setting $\mathcal{A}=\mathcal{A}(t)$ for ease of notation), if $\rho \geqslant \bar{\rho}(t)$,

$$
\begin{aligned}
\frac{t}{\rho^{p}} \frac{\mathcal{K}\left(E_{0}\right)}{E_{0}} \leqslant \frac{t}{\rho^{p}} \frac{\mathcal{K}\left[\gamma C^{-p-N} \xi\left(\mathcal{A}^{\frac{p+\theta-1}{p}}\right)\right]}{\gamma C^{-p-N} \xi\left(\mathcal{A}^{\frac{p+\theta-1}{p}}\right)} \leqslant \gamma \frac{t}{C^{(p+N) \Lambda} \rho^{p}} \frac{\mathcal{K}\left[\xi\left(\mathcal{A}^{\frac{p+\theta-1}{p}}\right)\right]}{\xi\left(\mathcal{A}^{\frac{p+\theta-1}{p}}\right)} \\
\leqslant \frac{\gamma}{C^{p+(p+N) \Lambda}}\left[\frac{\mathcal{A}^{p}}{\mathcal{D}(\mathcal{A})^{p-1} \varphi(\mathcal{A})}\right]^{1-\theta} \leqslant \frac{\gamma}{C^{p+(p+N) \Lambda}} .
\end{aligned}
$$


Therefore (5.12) is satisfied when $\rho \geqslant \bar{\rho}(t)$, provided $C$ is chosen large enough in the definition of $\bar{\rho}(t)$. The proof of estimate (1.22) is concluded.

Finally, we can prove the bound below for $Z(t)$ in (1.22) by observing that the estimate

$$
b\left(\|u(\cdot, t)\|_{\infty, \mathbb{R}^{N}}\right) Z(t)^{N} \geqslant \gamma_{0}\left|\int_{\mathbb{R}^{N}} v_{0}(x) \mathrm{d} x\right|
$$

follows from conservation of mass, and then substituting in it the sup bound (1.21). Note that here we look directly at $u$ of general sign.

\section{The thin-film equation}

In this section we perform an a priori investigation of the problem (1.1)-(1.4). More exactly, we look at solutions in a class defined by certain approximation properties, which are essentially the same usually considered in the literature on (1.1) with power $f$ (see (H) below). Thus, our method actually provides a complete proof of finite speed of propagation at least in that case; the main purpose of this section is to show that its scope is more general, even in the setting of the thin-film equation. For example, we note that functions given by

$$
f(s)=s^{a}|\ln s|^{q}, \quad 0<s \ll 1,
$$

meet all the requirements we stipulate below on $f$, if $3>a>2, q \in \mathbb{R}$.

Note that, denoting $\Omega=(-R, R)$, under the assumptions

$$
f \in C^{1+\alpha}(\mathbb{R}), \quad f(0)=0, \quad f(s)>0, s>0 ; \quad u_{0} \in W^{1,2}(\Omega), \quad u_{0} \geqslant 0,
$$

we can prove existence of a weak solution following the method of [16]. We use here the following definition.

Definition 6.1 A function $u \in C\left(\bar{Q}_{T}\right) \cap L^{\infty}\left(0, T ; W^{1,2}(\Omega)\right)$ is a weak solution of problem (1.1)-(1.4) if $u_{t}, u_{x}, u_{x x}, u_{x x x}, u_{x x x x}$ belong to $C(P)$, and $\sqrt{f(|u|)} u_{x x x} \in L^{2}(P)$, where $P=$ $\bar{Q}_{T} \backslash[\{u=0\} \cup\{t=0\}]$. Moreover, we require

$$
\iint_{Q_{T}} u \varphi_{t} \mathrm{~d} x \mathrm{~d} t+\iint_{P} f(|u|) u_{x x x} \varphi_{x} \mathrm{~d} x \mathrm{~d} t=0
$$

for all Lipschitz-continuous functions $\varphi$ with $\varphi \equiv 0$ near $t=0$ and $t=T$. The initial data are taken in a suitable pointwise sense, and (1.3) is also satisfied pointwise where $u( \pm R, t) \neq 0$. Moreover, $u_{x}(\cdot, t) \rightarrow u_{0 x}$ in $L^{2}(\Omega)$ as $t \rightarrow 0$.

If, additionally, $u(\cdot, t) \in C^{1}([-R, R])$ for almost every $t>0$, we say that $u$ is a strong solution.

As in [16], we prove the conservation law

$$
\int_{-R}^{R} u(x, t) \mathrm{d} x=\int_{-R}^{R} u_{0}(x) \mathrm{d} x, \quad t>0 .
$$

Note that below we work only with non-negative solutions, so that (6.4) becomes in fact an $L^{1}$ estimate. 
A necessary tool in our approach are certain inequalities of Bernis' type (see (6.9) below), whose proof in a non-power setting relies on the assumption

$$
\frac{A}{s} \geqslant \frac{f^{\prime}(s)}{f(s)} \geqslant \frac{B}{s}, \quad \text { for all } s>0, \text { and given } A>B>1 .
$$

If we assume $3>A>B>2$ we can prove in an elementary way, for all $s>0$,

$$
\begin{array}{cc}
G_{1}(s):=\int_{s}^{\infty} \frac{f(\tau)}{\tau^{4}} \mathrm{~d} \tau<\infty, & G_{2}(s):=\int_{0}^{s} G_{1}(\tau) \mathrm{d} \tau<\infty ; \\
\gamma_{0} f(s) \leqslant G_{2}(s) s^{2} \leqslant \gamma f(s), & \gamma_{0} f(s) \leqslant G_{3}(s) s \leqslant \gamma f(s),
\end{array}
$$

where $G_{3}(s):=\int_{0}^{s} G_{2}(\tau) \mathrm{d} \tau$. Alternatively, we can just require $A>B>1$, and directly assume (6.6), (6.7). Note that if $f(s)=s^{n}$, then (6.6) is equivalent to $2<n<3$. Here and below $\gamma, \gamma_{0}$ denote constants depending only on $A, B$. In the following we therefore assume that

$$
\text { either (i) } 3>A>B>2 \text {, or (ii) } A>B>1 \text { and (6.6), (6.7) hold. }
$$
0

Under the assumptions above, one can prove for all positive $v \in C^{3}[a, b]$, with $v^{\prime}(a)=v^{\prime}(b)=$

$$
\begin{aligned}
& \int_{a}^{b} G_{3}(v(x))\left|v^{\prime \prime}(x)\right|^{3} \mathrm{~d} x+\int_{a}^{b} \frac{f(v(x))}{v(x)^{4}}\left|v^{\prime}(x)\right|^{6} \mathrm{~d} x \\
& \quad \leqslant \gamma \int_{a}^{b} \frac{v(x)^{4}}{f(v(x))} G_{2}(v(x))^{2}\left|v^{\prime \prime \prime}(x)\right|^{2} \mathrm{~d} x \leqslant \gamma \int_{a}^{b} f(v(x))\left|v^{\prime \prime \prime}(x)\right|^{2} \mathrm{~d} x .
\end{aligned}
$$

The proof follows closely the lines of [13], and we therefore omit it.

Similarly, we confine ourselves to some comments on the proof of inequality (6.10), which will be the starting point of our argument:

$$
\begin{aligned}
& \sup _{0<\tau<t} \int_{-R}^{R}\left|\left(u \eta^{\frac{B+2}{2}}\right)_{x}\right|^{2} \mathrm{~d} x+\iint_{Q_{t}^{+}} \mathcal{F}[u \eta] \mathrm{d} x \mathrm{~d} \tau \leqslant \varepsilon \iint_{Q_{t}^{+} \cap\{\eta>0\}} \mathcal{F}[u] \mathrm{d} x \mathrm{~d} \tau \\
& +\gamma_{\varepsilon}\left[\left\|\eta_{x x x}\right\|_{\infty}^{2}+\left\|\eta_{x x}\right\|_{\infty}^{3}+\left\|\eta_{x}\right\|_{\infty}^{6}\right] \iint_{Q_{t} \cap\{\eta>0\}} f(u) u^{2} \mathrm{~d} x \mathrm{~d} \tau+\int_{-R}^{R}\left|\left(u_{0} \eta^{\frac{B+2}{2}}\right)_{x}\right|^{2} \mathrm{~d} x .
\end{aligned}
$$

Here $\eta \in C_{0}^{3}(-R, R)$ is any non-negative cutoff function, and $\varepsilon \in(0,1)$ can be chosen arbitrarily. Moreover, we denote

$$
Q_{t}^{+}=[(-R, R) \times(0, t)] \cap\{u>0\}, \quad \mathcal{F}[v]=f(v)\left|v_{x x x}\right|^{2}+\frac{f(v)}{v}\left|v_{x x}\right|^{3}+\frac{f(v)}{v^{4}}\left|v_{x}\right|^{6},
$$

for any sufficiently smooth positive function $v$. The proof of (6.10) is, as pointed out in [24] (when $f$ is a power), a consequence of Bernis' inequalities (i.e. (6.9) in our case), when $u(\cdot, t)$ is strictly positive a.e. $t>0$. It is a known fact that, when $f$ is a power, $2<n<3$, a solution to the original problem can be approximated by solutions of this kind, see $[10,14]$. Let us also remark that the approximation of $u$ with positive solutions has been proven of crucial relevance in the theory 
of the thin-film equation; indeed different approximation procedures select different solutions with different qualitative behaviour (see [10]). We set ourselves in the framework by now well known in the case of power nonlinearities, that is we directly assume:

$(\mathrm{H})$ a strong solution $u$ can be found as the uniform limit of strong solutions $u_{\delta}$, as $\delta \rightarrow$

0 , corresponding to initial data $u_{0 \delta}>0$ and such that $u_{\delta}(\cdot, t)>0$ a.e. $t>0$. Moreover $f\left(u_{\delta}\right)^{1 / 2} u_{\delta x x x} \rightarrow f(u)^{1 / 2} u_{x x x} \chi_{P}$ weakly in $L^{2}\left(Q_{T}\right)$; here $\chi_{P}$ is the characteristic function of the set $P$ defined above. We also stipulate that $f\left(u_{\delta}\right)^{1 / 2} u_{\delta x x} \rightarrow f(u)^{1 / 2} u_{x x} \chi_{P}$ strongly in $L^{2}\left(Q_{T}\right)$, and $u_{\delta x} \rightarrow u_{x}$ strongly in $L^{4}\left(Q_{T}\right)$. In turn each $u_{\delta}$ can be similarly approximated with a sequence $\left\{u_{\delta}^{\varepsilon}\right\}_{\varepsilon}$ of smooth positive solutions to nondegenerate parabolic equations.

In fact [14], essentially, deals with the convergence of each sequence $\left\{u_{\delta}^{\varepsilon}\right\}_{\varepsilon}$, but the relevant methods and results carry over immediately to the sequence $\left\{u_{\delta}\right\}_{\delta}$ itself (as a version of formula (3.5) in [14] still holds for $u_{\delta}$ ).

Lemma 6.1 Assume (H), and that $f$ satisfies (6.5) and (6.8). Then (6.10) holds for the solutions $u_{\delta}$ of problem (1.1)-(1.4) (with initial data $u_{0 \delta}$ ).

We refer the reader to [24] for more details on the proof of Lemma 6.1, which is indeed very similar to the case of a power function $f$ treated there, when we keep in mind (6.5)-(6.7).

In fact, what we need in our next Theorem are (6.10) and (6.2), (6.8). If we know a priori that (6.10) is fulfilled by $u$, the approximation steps in $(\mathrm{H})$ can be dispensed with.

THEOREM 6.1 Assume $u$ is a non-negative strong solution satisfying $(\mathrm{H})$, and that (6.5), (6.8) hold.

Then if $\operatorname{supp} u_{0} \subset\left[-r_{0}, r_{0}\right]$, we have that for $t>0 \operatorname{supp} u(\cdot, t) \subset[-z(t), z(t)]$, where $z(t)$ is defined by

$$
\frac{z(t)^{4}}{t}=C f\left(\frac{\left\|u_{0}\right\|_{1, \Omega}}{z(t)}\right)
$$

or by $z(t)=4 r_{0}$ if the solution $z(t)$ of $(6.11)$ is less than $4 r_{0}$ (i.e. for small $t$ ). Here $C$ depends on $A, B$ only.

Note that (6.11) is actually sharp; for example, when $f(s)=s^{n}, 2<n<3$ it gives the bound

$$
z(t) \leqslant \gamma\left\|u_{0}\right\|_{1}^{\frac{n}{n+4}} t^{\frac{1}{n+4}}, \quad \text { for large } t,
$$

which is known to be optimal, see [17]. More generally, we can prove the following result about the optimality of our estimate. Let us also recall that $(\mathrm{H})$ is known when $f$ is a power. Even in this case the next Proposition seems new.

Proposition 6.1 Define

$$
r(t)=\sup \{|x| \mid u(x, t)>0\} .
$$

Then, under the same assumptions of Theorem 6.1, and additionally stipulating $A<4$, we have (as long as $\left.4 r_{0}<z(t)<R\right)$

$$
r(t) \geqslant \gamma_{0} z(t)
$$

where $\gamma_{0}$ depends only on $A, B$, but not on $u, T, R$.

Clearly, Proposition 6.1 cannot hold for the solutions with stationary support constructed in [10]: indeed, those solutions are not the limit of $u_{\delta}$ as in $(\mathrm{H})$, but rather of solutions vanishing at some point $x$ for all positive times. 
Proof of Theorem 6.1. 1st step: a new energy inequality. We bound first supp $u$ on the right, i.e. we work with $x>0$. Let us define the intervals

$$
A_{0}=\left\{\left(1-\theta_{1}\right) \rho<x<\left(1+\theta_{1}\right) \rho\right\}, \quad A_{\infty}=\left\{\left(1-\theta_{2}\right) \rho<x<\left(1+\theta_{2}\right) \rho\right\},
$$

for a $\rho>4 r_{0}$, and $0<\theta_{1}<\theta_{2}<\frac{1}{2}$. Choose $\eta$ as a standard cutoff function in $A_{\infty}$, with $\eta \equiv 1$ in $A_{0}$; we may assume

$$
\left\|\eta_{x x x}\right\|_{\infty}^{2}+\left\|\eta_{x x}\right\|_{\infty}^{3}+\left\|\eta_{x}\right\|_{\infty}^{6} \leqslant \gamma(\theta \rho)^{-6} .
$$

In this proof we skip several iteration processes, which can be carried out rigorously as shown above. We also refrain from changing domains with each iteration process, formally referring to the pair $A_{0}, A_{\infty}$ in the whole proof (excepting the very last iteration, see (6.32)).

It follows from Poincaré's inequality that

$$
\int_{-R}^{R}\left|\left(u_{\delta} \eta^{\frac{B+2}{2}}\right)_{x}\right|^{2} \mathrm{~d} x \geqslant \frac{\gamma_{0}}{\rho^{2}} \int_{-R}^{R} u_{\delta}^{2} \eta^{B+2} \mathrm{~d} x .
$$

Let us write (6.10) for $u_{\delta}$. A standard iteration procedure, together with (6.14), now yields (when followed by the limit $\delta \rightarrow 0$ )

$$
\sup _{0<\tau<t} \frac{1}{\rho^{2}} \int_{A_{0}(\tau)} u^{2} \mathrm{~d} x+\int_{0}^{t} \int_{A_{0}^{+}} \mathcal{F}[u] \mathrm{d} x \mathrm{~d} \tau \leqslant \frac{\gamma}{(\theta \rho)^{6}} \int_{0}^{t} \int_{A_{\infty}} f(u) u^{2} \mathrm{~d} x \mathrm{~d} \tau .
$$

That is, we get (6.15) for each $u_{\delta}$, or, more exactly, (6.15) with an additional term containing the approximating initial datum on the right-hand side. This last term vanishes as $\delta \rightarrow 0$.

We denote $A_{0}^{+}=A_{0} \cap\{u>0\}$. Define the function

$$
h(s)=\int_{0}^{s} \frac{\mathrm{d} y}{y} \int_{0}^{y} \sqrt{f(\tau)} \mathrm{d} \tau, \quad s \geqslant 0 .
$$

A simple calculation (using the bound above in (6.5)) shows that

$$
\left|h^{\prime}(s)\right|^{2}+\left|h^{\prime \prime}(s)\right|^{2} s^{2}+\left|h^{\prime \prime \prime}(s)\right|^{2} s^{4} \leqslant \gamma f(s), \quad s>0 .
$$

Therefore it follows immediately from the definition of $\mathcal{F}[u]$ and Young's inequality that

$$
\left|h(u)_{x x x}\right|^{2} \leqslant \gamma \mathcal{F}[u] .
$$

Furthermore, using the bound below in (6.5), we show

$$
s^{2} f(s)^{2} \leqslant \gamma h(s)^{2}, \quad s \geqslant 0 .
$$

Hence, upon combining (6.15)-(6.19), we arrive at

$$
\sup _{0<\tau<t} \int_{A_{0}(\tau)} u^{2} \mathrm{~d} x+\rho^{2} \int_{0}^{t} \int_{A_{0}}\left|h(u)_{x x x}\right|^{2} \mathrm{~d} x \mathrm{~d} \tau \leqslant \frac{\gamma}{\theta^{6} \rho^{4}} \int_{0}^{t} \int_{A_{\infty}} h(u)^{2} \mathrm{~d} x \mathrm{~d} \tau .
$$

Next define

$$
v=h(u), \quad \varphi_{1}(v)=\left[h^{-1}(v)\right]^{2},
$$


and obtain the new energy inequality

$$
\sup _{0<\tau<t} \int_{A_{0}(\tau)} \varphi_{1}(v) \mathrm{d} x+\rho^{2} \int_{0}^{t} \int_{A_{0}}\left|v_{x x x}\right|^{2} \mathrm{~d} x \mathrm{~d} \tau \leqslant \frac{\gamma}{\theta^{6} \rho^{4}} \int_{0}^{t} \int_{A_{\infty}} v^{2} \mathrm{~d} x \mathrm{~d} \tau .
$$

2nd step: a preliminary estimate of $z(t)$ via a Dini function. This part of the proof is essentially a repetition of the proof given in Section 4.

Define for $s \geqslant 0$

$$
\begin{gathered}
\mathcal{D}_{1}(s):=\int_{0}^{s} \frac{\mathrm{d} \tau}{\varphi_{1}^{\prime}(\tau)}=\int_{0}^{s} \frac{h^{\prime}\left(h^{-1}(\tau)\right)}{2 h^{-1}(\tau)} \mathrm{d} \tau \leqslant \gamma \int_{0}^{s} \frac{\sqrt{f\left(h^{-1}(\tau)\right)}}{h^{-1}(\tau)} \mathrm{d} \tau \\
=\gamma \int_{0}^{h^{-1}(s)} \frac{\sqrt{f(t)}}{t} h^{\prime}(t) \mathrm{d} t \leqslant \gamma \int_{0}^{h^{-1}(s)} \frac{f(t)}{t} \mathrm{~d} t<\infty,
\end{gathered}
$$

where we have made use of the bound (6.17) for $h^{\prime}$, and again of the bound below in (6.5): this last bound in fact implies $f(s) \leqslant s^{B} f(1), 0<s<1$. Note that

$$
s^{2} \leqslant \mathcal{D}_{1}(s) \varphi_{1}(s), \quad s>0,
$$

follows as (3.4) from an application of Hölder's inequality. Moreover, (6.5) and the explicit form of $h$ allow one to prove easily for all $s>0$

$$
\mathcal{D}_{1}\left(\varphi_{1}^{-1}(a s)\right) \leqslant a^{q_{1}} \mathcal{D}_{1}\left(\varphi_{1}^{-1}(s)\right), \quad a>1 ; \quad \mathcal{D}_{1}\left(\varphi_{1}^{-1}(\alpha s)\right) \leqslant \alpha^{q_{2}} \mathcal{D}_{1}\left(\varphi_{1}^{-1}(s)\right), \quad \alpha<1,
$$

where we have set $q_{1}=A / 2, q_{2}=B / 2$. The estimates (6.24), (6.25) are all we need for the proof of Lemma 3.1 to remain valid in the present setting. Indeed, (6.24) is used in (the analogues of) (3.15), (3.16), and (6.25) in (3.18). In the case at hand the embedding is

$$
\begin{aligned}
\int_{a}^{b} v(x)^{2} \mathrm{~d} x \leqslant \varepsilon(b-a)^{6} & \int_{a}^{b}\left|v_{x x x}(x)\right|^{2} \mathrm{~d} x \\
& +\gamma \varepsilon^{-\omega} \int_{a}^{b} \varphi_{1}(v(x)) \mathrm{d} x \mathcal{D}_{1}\left(\varphi_{1}^{-1}\left(\frac{1}{b-a} \int_{a}^{b} \varphi_{1}(v(x)) \mathrm{d} x\right)\right),
\end{aligned}
$$

where we assume $b>a, v \in W^{3,2}(a, b), \varphi_{1}(v) \in L^{1}(a, b)$; here $\varepsilon \in(0,1)$ is arbitrary and $\omega=\omega(A, B)$.

The right-hand side of (6.22) can then be bound above by

$$
\frac{\varepsilon \rho^{2}}{\theta^{6}} \int_{0}^{t} \int_{A_{\infty}}\left|v_{x x x}\right|^{2} \mathrm{~d} x \mathrm{~d} \tau+\frac{\gamma \varepsilon^{-\omega} t}{\theta^{6} \rho^{4}} \sup _{0<\tau<t} \int_{A_{\infty}(\tau)} \varphi_{1}(v) \mathrm{d} x \mathcal{D}_{1}\left(\varphi_{1}^{-1}\left(\sup _{0<\tau<t} \frac{1}{\rho} \int_{A_{\infty}(\tau)} \varphi_{1}(v) \mathrm{d} x\right)\right) .
$$

Selecting a small enough $\varepsilon$, and applying a suitable interpolation procedure, we get

$$
\begin{aligned}
\sup _{0<\tau<t} \int_{A_{0}(\tau)} & \varphi_{1}(v) \mathrm{d} x+\rho^{2} \int_{0}^{t} \int_{A_{0}}\left|v_{x x x}\right|^{2} \mathrm{~d} x \mathrm{~d} \tau \\
& \leqslant \frac{\gamma t}{\theta^{6 \omega+6} \rho^{4}} \sup _{0<\tau<t} \int_{A_{\infty}(\tau)} \varphi_{1}(v) \mathrm{d} x \mathcal{D}_{1}\left(\varphi_{1}^{-1}\left(\sup _{0<\tau<t} \frac{1}{\rho} \int_{A_{\infty}(\tau)} \varphi_{1}(v) \mathrm{d} x\right)\right) .
\end{aligned}
$$


Define, for a sequence of intervals $I_{n+1} \subset I_{n}$ interpolating between $A_{0}$ and $A_{\infty}$ (see (4.8))

$$
Y_{n}=\sup _{0<\tau<t} \frac{1}{\rho} \int_{I_{n}(\tau)} \varphi_{1}(v) \mathrm{d} x .
$$

Thus, applying (6.27) to the pair $I_{n+1} \subset I_{n}$, we find, for a suitable $\gamma>1$,

$$
Y_{n+1} \leqslant \gamma^{n} \frac{t}{\rho^{4}} Y_{n} \mathcal{D}_{1}\left(\varphi_{1}\left(Y_{n}\right)\right)
$$

Reasoning as in (4.10), we obtain $Y_{n} \rightarrow 0, n \rightarrow \infty$ if

$$
\frac{t}{\rho^{4}} \mathcal{D}_{1}\left(\varphi_{1}\left(Y_{0}\right)\right) \leqslant \gamma_{0}
$$

We exploit (6.25) again here, analogously to what we did in Section 4. Obviously, (6.29) gives a first estimate of $z(t)$.

3rd step: refining the estimate. This part is meaningful in the case of the thin-film equation, where we have $L^{1}$ estimates of the non-negative solution available. We start again from (6.22), and prove a new bound for $Y_{0}$. Combining this estimate with (6.29) we arrive at the final result.

Recall $v=h(u)$, and define

$$
\varphi_{0}(v):=h^{-1}(v)=u, \quad \mathcal{D}_{0}(s):=\int_{0}^{s} \frac{\mathrm{d} \tau}{\varphi_{0}^{\prime}(\tau)}<\infty .
$$

The finiteness of the Dini function $\mathcal{D}_{0}(s)$ can be proven as in (6.23) above, taking into account (6.17). Moreover, (6.24) and (6.25) are still satisfied when we formally replace $\mathcal{D}_{1}$ with $\mathcal{D}_{0}, \varphi_{1}$ with $\varphi_{0}$, and set $q_{1}=1+A, q_{2}=1+B$. This is again, as above, an elementary consequence of our assumptions on $f$. Thus, Lemma 3.1 gives

$$
\begin{aligned}
\int_{a}^{b} v(x)^{2} \mathrm{~d} x \leqslant \varepsilon(b-a)^{6} & \int_{a}^{b}\left|v_{x x x}(x)\right|^{2} \mathrm{~d} x \\
& +\gamma \varepsilon^{-\omega} \int_{a}^{b} \varphi_{0}(v(x)) \mathrm{d} x \mathcal{D}_{0}\left(\varphi_{0}^{-1}\left(\frac{1}{b-a} \int_{a}^{b} \varphi_{0}(v(x)) \mathrm{d} x\right)\right),
\end{aligned}
$$

where we assume $b>a, v \in W^{3,2}(a, b), \varphi_{0}(v) \in L^{1}(a, b)$; here $\varepsilon \in(0,1)$ is arbitrary and $\omega=\omega(A, B)$. The integral of $v^{2}=h(u)^{2}$ in (6.22) can be therefore majorized by

$$
\varepsilon \rho^{6} \int_{0}^{t} \int_{A_{\infty}}\left|v_{x x x}\right|^{2} \mathrm{~d} x \mathrm{~d} \tau+\varepsilon^{-\omega} t \sup _{0<\tau<t} \int_{A_{\infty}(\tau)} u \mathrm{~d} x \mathcal{D}_{0}\left(h\left(\sup _{0<\tau<t} \frac{1}{\rho} \int_{A_{\infty}(\tau)} u \mathrm{~d} x\right)\right)
$$

(we recall that $\varphi_{0}=h^{-1}$ ). Then a final iteration procedure allows one to absorb the term containing $v_{x x x}$ in the resulting inequality, yielding for $Y_{0}$ defined as in (6.28)

$$
Y_{0}=\sup _{0<\tau<t} \frac{1}{\rho} \int_{A_{\infty}(\tau)} u^{2} \mathrm{~d} x \leqslant \frac{\gamma t}{\rho^{4}} \sup _{0<\tau<t} \frac{1}{\rho} \int_{A_{\infty}^{\prime}(\tau)} u \mathrm{~d} x \mathcal{D}_{0}\left(h\left(\sup _{0<\tau<t} \frac{1}{\rho} \int_{A_{\infty}^{\prime}(\tau)} u \mathrm{~d} x\right)\right) .
$$


Here $A_{\infty}^{\prime}$ is an interval strictly containing $A_{\infty}$, as required by the aforementioned iteration process. Employing the mass conservation law (6.4) we obtain

$$
Y_{0} \leqslant \gamma \frac{t}{\rho^{5}}\left\|u_{0}\right\|_{1, \Omega} \mathcal{D}_{0}\left(h\left(\frac{\left\|u_{0}\right\|_{1, \Omega}}{\rho}\right)\right) .
$$

Substituting this bound in (6.29), we conclude that $Y_{n} \rightarrow 0, n \rightarrow \infty$, i.e. $u(\rho, t)=0$, if $\rho$ is chosen so as

$$
\frac{t}{\rho^{4}} \mathcal{D}_{1}\left[\varphi^{-1}\left[\frac{t}{\rho^{5}}\left\|u_{0}\right\|_{1, \Omega} \mathcal{D}_{0}\left(h\left(\frac{\left\|u_{0}\right\|_{1, \Omega}}{\rho}\right)\right)\right]\right] \leqslant \gamma_{0},
$$

for a suitable $\gamma_{0}$. Next we take into account the inequalities

$$
\mathcal{D}_{0}(h(s)) \leqslant \gamma s f(s), \quad \mathcal{D}_{1}\left(\varphi_{1}^{-1}(s)\right) \leqslant \gamma f(\sqrt{s}), \quad s>0,
$$

which can be proven using (6.5), (6.17) and the explicit definitions of the involved functions. Thus (6.33) is implied by

$$
J(\rho, t):=\frac{t}{\rho^{4}} f\left\{\frac{\left\|u_{0}\right\|_{1, \Omega}}{\rho^{3}}\left[t f\left(\frac{\left\|u_{0}\right\|_{1, \Omega}}{\rho}\right)\right]^{1 / 2}\right\} \leqslant \gamma_{0}<1 .
$$

Let us define now $z(t)$ by

$$
\frac{z(t)^{4}}{t}=C f\left(\frac{\left\|u_{0}\right\|_{1, \Omega}}{z(t)}\right)
$$

where $C>1$ is to be chosen presently. If $\rho \geqslant z(t)$, we have, by definition of $z(t)$,

$$
\begin{aligned}
& J(\rho, t) \leqslant J(z(t), t)=\frac{t}{z(t)^{4}} f\left\{\frac{\left\|u_{0}\right\|_{1, \Omega}}{z(t)^{3}}\left[t \frac{z(t)^{4}}{C t}\right]^{1 / 2}\right\} \\
& =\frac{t}{z(t)^{4}} f\left\{\frac{\left\|u_{0}\right\|_{1, \Omega}}{C^{1 / 2} z(t)}\right\} \leqslant \frac{t}{z(t)^{4}} f\left\{\frac{\left\|u_{0}\right\|_{1, \Omega}}{z(t)}\right\}=\frac{1}{C} \leqslant \gamma_{0},
\end{aligned}
$$

if we choose $C=1 / \gamma_{0}$. The theorem follows immediately, when we note that for $x<0$ the proof can be clearly reproduced without changes.

Proof of Proposition 6.1 Let $u_{\delta}$ be the approximating sequence in (H). We may make use of (6.9) and of Hölder's inequality to get at almost each time level $t>0$

$$
\begin{aligned}
\int_{-R}^{R} u_{\delta x}^{2} \mathrm{~d} x \leqslant\left[\int_{-R}^{R} \frac{f\left(u_{\delta}\right)}{u_{\delta}^{4}}\left|u_{\delta x}\right|^{6} \mathrm{~d} x\right]^{\frac{1}{3}}\left[\int_{-R}^{R} \frac{u_{\delta}^{2}}{\sqrt{f\left(u_{\delta}\right)}} \mathrm{d} x\right]^{\frac{2}{3}} \\
\leqslant \gamma\left[\int_{-R}^{R} f\left(u_{\delta}\right)\left|u_{\delta x x x}\right|^{2} \mathrm{~d} x\right]^{\frac{1}{3}}\left[\int_{-R}^{R} \frac{u_{\delta}^{2}}{\sqrt{f\left(u_{\delta}\right)}} \mathrm{d} x\right]^{\frac{2}{3}} .
\end{aligned}
$$

The function $r(t)$ defined in the statement measures the actual size of the support of the solution $u$, while the function $z(t) \geqslant r(t)$ defined as in Theorem 6.1 is an estimate of it. Recall that we assume 
in this proof $z(t)<R$. In the following we denote $I(t)=(-r(t), r(t)), K(t)=(-z(t), z(t))$. By elementary calculus we have

$$
\left\|u_{\delta}(\cdot, t)\right\|_{\infty, \Omega} \leqslant c_{1 \delta}+(2 r(t))^{\frac{1}{2}}\left(\int_{-R}^{R} u_{\delta x}^{2} \mathrm{~d} x\right)^{\frac{1}{2}},
$$

where

$$
c_{1 \delta}:=\max \left\{u_{\delta}(x, t)|0 \leqslant t \leqslant T, r(t) \leqslant| x \mid \leqslant R\right\} \rightarrow 0, \quad \text { as } \delta \rightarrow 0,
$$

owing to the uniform convergence $u_{\delta} \rightarrow u$. Obviously (6.36) still holds if we substitute $r$ with $z$ there. Keeping in mind that $A<4$, we check that $s \mapsto s^{4} / f(s)$ is increasing. Then

$$
\begin{aligned}
& \int_{-R}^{R} \frac{u_{\delta}^{2}}{\sqrt{f\left(u_{\delta}\right)}} \mathrm{d} x=\int_{-z(t)}^{z(t)} \frac{u_{\delta}^{2}}{\sqrt{f\left(u_{\delta}\right)}} \mathrm{d} x+\int_{|x|>z(t)} \frac{u_{\delta}^{2}}{\sqrt{f\left(u_{\delta}\right)}} \mathrm{d} x \\
& \leqslant c_{2 \delta}+2 z(t) \frac{\left\|u_{\delta}(\cdot, t)\right\|_{\infty, K(t)}^{2}}{\sqrt{f\left(\left\|u_{\delta}(\cdot, t)\right\|_{\infty, K(t)}\right)}} \leqslant c_{2 \delta}+\gamma z(t) \frac{\left\|u_{\delta}(\cdot, t)\right\|_{\infty, I(t)}^{2}+\left\|u_{\delta}(\cdot, t)\right\|_{\infty, K(t) \backslash I(t)}^{2}}{\sqrt{f\left(\left\|u_{\delta}(\cdot, t)\right\|_{\infty, K(t))}\right.}} \\
& \leqslant c_{4 \delta}(t)+\gamma z(t) \frac{\left\|u_{\delta}(\cdot, t)\right\|_{\infty, I(t)}^{2}}{\sqrt{f\left(\left\|u_{\delta}(\cdot, t)\right\|_{\infty, I(t))}\right.}},
\end{aligned}
$$

where $c_{2 \delta}$ denotes the third integral in (6.37), and

$$
c_{4 \delta}(t)=c_{2 \delta}+\gamma \frac{R\left\|u_{\delta}(\cdot, t)\right\|_{\infty, K(t) \backslash I(t)}^{2}}{\sqrt{f\left(\left\|u_{\delta}(\cdot, t)\right\|_{\infty, K(t)}\right.}} .
$$

Clearly $c_{4 \delta}(t) \rightarrow 0$ as $\delta \rightarrow 0$, for all $t>0$, since $u_{\delta}$ converges to a function with positive maximum, at each time level (this follows from conservation of mass). We now make use of (6.36) (with $r$ replaced with $z$ ), to obtain

$$
\begin{aligned}
\int_{-R}^{R} \frac{u_{\delta}^{2}}{\sqrt{f\left(u_{\delta}\right)}} \mathrm{d} x \leqslant c_{4 \delta}(t)+\gamma z(t) & \frac{c_{1 \delta}^{2}+z(t) \int_{-R}^{R} u_{\delta x}^{2} \mathrm{~d} x}{f\left(c_{1 \delta}+z(t)^{\frac{1}{2}}\left(\int_{-R}^{R} u_{\delta x}^{2} \mathrm{~d} x\right)^{\frac{1}{2}}\right)^{\frac{1}{2}}} \\
& \leqslant c_{5 \delta}(t)+\gamma \frac{z(t)^{2} \int_{-R}^{R} u_{\delta x}^{2} \mathrm{~d} x}{f\left(z(t)^{\frac{1}{2}}\left(\int_{-R}^{R} u_{\delta x}^{2} \mathrm{~d} x\right)^{\frac{1}{2}}\right)^{\frac{1}{2}}}=: J_{1 \delta}(t),
\end{aligned}
$$

where

$$
c_{5 \delta}(t)=c_{4 \delta}(t)+\gamma \frac{R c_{1 \delta}^{2}}{\sqrt{f\left(c_{1 \delta}\right)}} \rightarrow 0, \quad \text { as } \delta \rightarrow 0 .
$$

From (6.35) and (6.38) we get

$$
\int_{-R}^{R} f\left(u_{\delta}\right)\left|u_{\delta x x x}\right|^{2} \mathrm{~d} x \geqslant \gamma_{0}\left(\int_{-R}^{R} u_{\delta x}^{2} \mathrm{~d} x\right)^{3} J_{1 \delta}(t)^{-2}=: J_{2 \delta}(t) .
$$


Note that the strong convergence $u_{\delta x} \rightarrow u_{x}$ in $L^{4}\left(Q_{T}\right)$ implies that we may assume, perhaps extracting a subsequence,

$$
u_{\delta x}(\cdot, t) \rightarrow u_{x}(\cdot, t), \quad \text { in } L^{4}(-R, R), \text { a.e. } t>0 .
$$

Thus, we have as $\delta \rightarrow 0$, for almost every $t>0$,

$$
J_{2 \delta}(t) \rightarrow \gamma \frac{1}{z(t)^{4}} f\left(z(t)^{\frac{1}{2}}\left(\int_{-R}^{R} u_{x}^{2} \mathrm{~d} x\right)^{\frac{1}{2}}\right) \int_{-R}^{R} u_{x}^{2} \mathrm{~d} x .
$$

On multiplying the equation by $u_{\delta x x}$ and integrating by parts we get, using (6.39),

$$
\begin{aligned}
\int_{-R}^{R} u_{\delta x}(x, t)^{2} \mathrm{~d} x \leqslant-2 \iint_{Q_{t} \cap\left\{\tau>t_{0}\right\}} f\left(u_{\delta}\right)\left|u_{\delta x x x}\right|^{2} \mathrm{~d} x \mathrm{~d} \tau & +\int_{-R}^{R} u_{\delta x}\left(x, t_{0}\right)^{2} \mathrm{~d} x \\
& \leqslant-2 \int_{t_{0}}^{t} J_{2 \delta}(\tau) \mathrm{d} \tau+\int_{-R}^{R} u_{\delta x}\left(x, t_{0}\right)^{2} \mathrm{~d} x
\end{aligned}
$$

for a.e. $t, t_{0}$, with $t>t_{0}>0$. (Here we exploit the possibility of approximating $u_{\delta}$ with regular strictly positive solutions $u_{\delta}^{\varepsilon}$ as in (H); strong convergence of first space derivatives takes place a.e. in $t$.) On letting $\delta \rightarrow 0$, we find

$$
\begin{aligned}
\int_{-R}^{R} u_{x}(x, t)^{2} \mathrm{~d} x \leqslant-\gamma_{1} \int_{t_{0}}^{t} \frac{1}{z(\tau)^{4}}\left(\int_{-R}^{R} u_{x}^{2} \mathrm{~d} x\right) f\left(z(\tau)^{\frac{1}{2}}\left(\int_{-R}^{R} u_{x}^{2} \mathrm{~d} x\right)^{\frac{1}{2}}\right) \mathrm{d} \tau & \\
& +\int_{-R}^{R} u_{x}\left(x, t_{0}\right)^{2} \mathrm{~d} x .
\end{aligned}
$$

We stress the fact the the above inequality holds for all $t>t_{0}, t, t_{0} \in I$, where $I \subset(0, T)$, and $|I|=T$. Then standard comparison results yield the bound $z(t) \int_{-R}^{R} u_{x}^{2} \mathrm{~d} x \leqslant y(t)^{2}$, where

$$
\left\{\begin{array}{l}
\frac{\mathrm{d}}{\mathrm{d} t}\left(\frac{y^{2}}{z}\right)=-\frac{\gamma_{1}}{z^{5}} f(y) y^{2} \\
y(0)^{2}=2 z(0) \int_{-R}^{R} u_{0 x}^{2} \mathrm{~d} x>0 .
\end{array}\right.
$$

We next proceed to estimate $y(t)$. The differential equation in (6.41) can be rewritten as

$$
y^{\prime}=\frac{z^{\prime} y}{2 z}-\frac{\gamma_{1}}{2 z^{4}} f(y) y .
$$

We distinguish between the two cases

$$
\begin{aligned}
& \frac{z^{\prime} y}{2 z} \geqslant \delta \frac{\gamma_{1}}{2 z^{4}} f(y) y \\
& \frac{z^{\prime} y}{2 z}<\delta \frac{\gamma_{1}}{2 z^{4}} f(y) y .
\end{aligned}
$$


Here $\delta \in(0,1)$ is selected so as

$$
\frac{\delta}{1-\delta}=\frac{B(4+A)}{2 A(4+B)}
$$

Define $\bar{t}=\sup \left\{t>0 \mid z(t)=4 r_{0}\right\}$; then for $t \geqslant \bar{t} z(t)$ is given by (6.11). Note that $z \in$ $A C_{\mathrm{loc}}(0, \infty)$, and that $z$ is in fact of class $C^{1}$ for $t \neq \bar{t}$.

Define also the sets

$$
S_{1}=\{t>\bar{t} \mid(6.43) \text { holds }\}, \quad S_{2}=\{t>\bar{t} \mid(6.44) \text { holds }\}=\operatorname{int} S_{2} .
$$

Note that, when $t>\bar{t}$, by differentiating the equality defining $z$, we find

$$
z^{\prime}\left(\frac{4 z^{3}}{t}+C f^{\prime}\left(\frac{\left\|u_{0}\right\|_{1, \Omega}}{z}\right) \frac{\left\|u_{0}\right\|_{1, \Omega}}{z^{2}}\right)=\frac{z^{4}}{t^{2}}, \quad \text { so that } \quad \frac{z(t)}{(4+A) t} \leqslant z^{\prime}(t) \leqslant \frac{z(t)}{(4+B) t},
$$

when we invoke (6.5). Hence, for all $t \in S_{1}$ we have

$$
f(y(t)) \leqslant \frac{1}{(4+B) \delta \gamma_{1}} \frac{z(t)^{4}}{t} .
$$

Let us now consider the case when $t \in S_{2}$; let $t_{0}$ be the least time such that $\left(t_{0}, t\right) \subset S_{2}$. Assume first that $t_{0}>\bar{t}$, so that $t_{0} \in S_{1}$. Clearly, in $\left(t_{0}, t\right)$ we have, owing to (6.42) and (6.44),

$$
y^{\prime}<-(1-\delta) \gamma_{1} \frac{f(y) y}{2 z^{4}} .
$$

A trivial integration by separation of variables, together with the inequalities

$$
\frac{\mathrm{d}}{\mathrm{d} y} \frac{1}{f(y)}=-\frac{f^{\prime}(y)}{f(y)^{2}} \leqslant-\frac{B}{f(y) y} ; \quad \frac{\mathrm{d}}{\mathrm{d} t} \frac{t}{z(t)^{4}} \leqslant \frac{A}{4+A} \frac{1}{z(t)^{4}}, \quad t>\bar{t}
$$

(the second one following from (6.45)), yield

$$
-\frac{1}{f(y(t))} \leqslant-\frac{1}{f\left(y\left(t_{0}\right)\right)}+(1-\delta) \gamma_{1} \frac{B(4+A)}{2 A} \frac{t_{0}}{z\left(t_{0}\right)^{4}}-(1-\delta) \gamma_{1} \frac{B(4+A)}{2 A} \frac{t}{z(t)^{4}} .
$$

We have also used $y(t)<y\left(t_{0}\right)$, which is an obvious consequence of (6.47). The sum of the first and second term on the right-hand side of this inequality is in fact nonpositive; indeed, recalling that (6.46) is in force for $t=t_{0} \in S_{1}$, we find

$$
\frac{1}{f\left(y\left(t_{0}\right)\right)} \geqslant \delta \gamma_{1}(4+B) \frac{t_{0}}{z\left(t_{0}\right)^{4}}=(1-\delta) \gamma_{1} \frac{4+A}{2 A} \frac{t_{0}}{z\left(t_{0}\right)^{4}},
$$

because of our definition of $\delta$. Then (6.48) gives at once

$$
f(y(t)) \leqslant \frac{2 A}{B(4+A)(1-\delta) \gamma_{1}} \frac{z(t)^{4}}{t} .
$$

The case $t \in S_{2}, t_{0}=\bar{t}$ is left to be considered. Clearly, (6.47) is then satisfied over $(0, t) \backslash\{\bar{t}\}$, if we keep in mind that $z^{\prime} \equiv 0$ for $t<\bar{t}$. Integrating this differential inequality over $(0, t)$, treating the 
term $y^{\prime} / f(y)$ as shown above, and exploiting now simply the nondecreasing character of $z$ (instead of (6.45)), we arrive at

$$
-\frac{1}{f(y(t))} \leqslant-\frac{1}{f(y(t))}+\frac{1}{f(y(0))} \leqslant-(1-\delta) \gamma_{1} B \frac{t}{2 z(t)^{4}} .
$$

Collecting the estimates (6.46), (6.49) and (6.50), we find the bound above

$$
f(y(t)) \leqslant \gamma \frac{z(t)^{4}}{t}, \quad \text { for all } t>\bar{t},
$$

and a suitable $\gamma$.

Thus Hölder's and Poincaré's inequalities give for $t>\bar{t}$

$$
\begin{aligned}
\left\|u_{0}\right\|_{1, \Omega} & =\int_{-R}^{R} u \mathrm{~d} x \leqslant \sqrt{2 r(t)}\left(\int_{-R}^{R} u^{2} \mathrm{~d} x\right)^{\frac{1}{2}} \\
& \leqslant \gamma \sqrt{r(t)} r(t)\left(\int_{-R}^{R} u_{x}^{2} \mathrm{~d} x\right)^{\frac{1}{2}} \leqslant \gamma \sqrt{\frac{r(t)}{z(t)}} r(t) y(t) \quad(\text { by }(6.51)) \\
& \leqslant \gamma \sqrt{\frac{r(t)}{z(t)}} r(t) f^{-1}\left(\frac{z(t)^{4}}{t}\right) \leqslant \gamma \sqrt{\frac{r(t)}{z(t)}} r(t) \frac{\left\|u_{0}\right\|_{1, \Omega}}{z(t)}
\end{aligned}
$$

where the last inequality follows from the definition of $z(t)$.

Proposition 6.1 is proven.

\section{Acknowledgements}

We are indebted to the referees for helpful comments on the presentation of the paper, and for pointing out the interest of the class of examples in (1.12). We also thank Dr Lorenzo Giacomelli for interesting talks on the thin film equation. DA is a member of the Italian GNFM, and is partially supported by MURST project 'Problemi a frontiera libera'.

\section{REFERENCES}

1. Alt, H. W. \& Luckhaus, S. Quasilinear elliptic-parabolic differential equations. Math. Z. 183, (1983) 311-341.

2. ANDREUCCI, D. $l_{\text {loc }}^{\infty}$-estimates for local solutions of degenerate parabolic equations. SIAM J. Math. Anal. 22, (1991) 138-145. Philadelphia PE (USA).

3. Andreucci, D. \& DiBenedetto, E. On the Cauchy problem and initial traces for a class of evolution equations with strongly nonlinear sources. Annali Sc. Normale Sup. Pisa 18, (1991) 363-441.

4. AndreucCI, D. \& TEDEe, A. F. A Fujita type result for a degenerate Neumann problem in domains with non compact boundary. J. Math. Anal. Appl. 231, (1999) 543-567.

5. Andreucci, D. \& Tedeev, A. F. Sharp estimates and finite speed of propagation for a Neumann problem in domains narrowing at infinity. Adv. Differ. Equ. 5, (2000) 833-860.

6. Antontsev, S. N. On the localization of solutions of nonlinear degenerate elliptic and parabolic equations. Sov. Math. Dokl. 24, (1981) 420-424.

7. Barenblatt, G. I. On self-similar motions of a compressible fluid in a porous medium. Prikl. Mat. Mech. 16, (1952) 679-698. 
8. BÉnilan, P. Personal communication.

9. BÉnilan, P. \& Wittbold, P. On mild and weak solutions of elliptic-parabolic problems. Adv. Differ. Equ. 1, (1996) 1053-1073.

10. Beretta, E., Bertsch, M., \& Dal Passo, R. Nonnegative solutions of a fourth-order nonlinear degenerate parabolic equation. Arch. Rat. Mech. Anal. 129, (1995) 175-200.

11. BERNIS, F. Existence results for doubly nonlinear higher order parabolic equations on unbounded domains. Mathematische Annalen 279, (1988) 373-394.

12. BERNIS, F. Qualitative properties for some nonlinear higher order degenerate parabolic equations. Houston J. Math. 14, (1988) 319-352.

13. BERNIS, F. Energy methods for higher order elliptic and parabolic problems. In: ANTONTSEV, S., DiAZ, J. I. \& ShmareV, S. (eds), Energy Methods in Continuum Mechanics. pp. 31-37. Kluwer, Dordrecht (1996).

14. BERnis, F. Finite speed of propagation and continuity of the interface for thin viscous flow. Adv. Differ. Equ. 1, (1996) 337-368.

15. Bernis, F. Finite speed of propagation for thin viscous flows when $2 \leqslant n<3$. C. R. Acad. Sci. Paris, Series I 322, (1996) 1169-1174.

16. Bernis, F. \& Friedman, A. Higher order nonlinear degenerate parabolic equations. J. Differ. Equ. 83 (1990) 179-206.

17. Bernis, F., Peletier, L. A., \& Williams, S. M. Source type solutions of a fourth order nonlinear degenerate parabolic equation. Nonlinear Anal. TMA 18, (1992) 217-234.

18. BERTOZZI, A. Symmetric singularity formation in lubrication-type equations for interface motion. SIAM J. Appl. Math. 56, (1996) 681-714.

19. Bertsch, M., Dal Passo, R., Garcke, H., \& Grün, G. The thin viscous flow equation in higher space dimension. Adv. Differ. Equ. 3, (1998) 417-440.

20. DiAZ, J. I. Solutions with compact support for some degenerate parabolic problems. Nonlinear. Anal. TMA 3, (1979) 831-847.

21. DiAZ, J. I. \& VERON, L. Local vanishing properties of solutions of elliptic and parabolic quasilinear equations. Trans. Am. Math. Soc. 290, (1985) 787-814.

22. DiBenedetto, E. Degenerate Parabolic Equations. Springer, New York, NY (1993).

23. Gilding, B. H. \& Kersner, R. A necessary and sufficient condition for finite speed of propagation in the theory of doubly nonlinear degenerate parabolic equations. Proc. R. Soc. Edinburgh A 126, (1996) 739-767.

24. Hulshof, J. \& Shishrov, A. The thin film equation with $2 \leqslant n<3$ : finite speed of propagation in terms of the $l^{1}$-norm. Adv. Differ. Equ. 3, (1998) 625-642.

25. Kalashnikov, A. S. Formation of singularities in solutions of the equation of non-stationary filtration. Zh. Vychisl. Mat. Mat. Fiz. 7, (1967) 440-444.

26. Kalashnikov, A. S. Some problems on the qualitative theory of non-linear degenerate second-order parabolic equations. Usp. Mat. Nauk 42, (1987) 135-176. (English translation in Russ. Math. Surv. 42 (1987), 169-222.)

27. Kalashnikov, A. S., Oleinik, O. A., \& Yui-Lin, C. The Cauchy problem and boundary value problems for equations of the type of non-stationary filtration. Izv. Akad. Nauk SSSR Ser. Mat. 22, (1958) 667-704.

28. Kalita, E. A. Compactness of the support and the nonexistence of singularities for nonlinear elliptic systems of arbitrary order. Sibirsk. Mat. Zh. 35, (1994) 327-339. (English Transl. Siberian Math. J. 35 (1994), 294-305.)

29. Ladyzhenskaja, O. A., Solonnikov, V. A., \& Ural'CeVA, N. N. Linear and quasilinear equations of parabolic type. Translations of Mathematical Monographs, vol. 23. American Mathematical Society, Providence, RI (1968).

30. Peletier, L. A. A necessary and sufficient condition for the existence of an interface in flow through porous media. Arch. Rat. Mech. Anal. 56, (1974) 183-190. 
31. RAO, M. \& REN, Z. Theory of orlicz spaces. Pure and Applied Mathematics. Marcel Dekker, New York, NY 146, (1991).

32. Shishkov, A. E. Evolution of the support of a solution with unbounded energy of a quasi-linear degenerate parabolic equation of arbitrary order. Mathematicheskii Sbornik 186, (1995) 151-172.

33. Shishkov, A. E. Propagation of perturbation in a singular Cauchy problem for degenerate quasilinear parabolic equations. Mathematicheskii Sbornik 187, (1996) 139-159.

34. SteIn, E. M. Singular Integrals and Differentiability Properties of Functions. Princeton University Press, Princeton, NJ (1970)

35. Watanabe, M. Solutions with compact support of the porous medium equation in arbitrary dimension. Proc. AMS 103, (1988) 149-152. 